Pacific Journal of Mathematics

CONVERGENCE FOR THE SQUARE ROOT OF THE POISSON 


\title{
CONVERGENCE FOR THE SQUARE ROOT OF THE POISSON KERNEL
}

\author{
Peter SJÖGREN
}

\begin{abstract}
Let $X$ be a symmetric space and $f$ an integrable function on its boundary $\partial X$. The 0 -Poisson integral $P_{0} f$ is the function on $X$ obtained by integrating $f$ against the square root of the Poisson kernel. We give Fatou theorems saying that the normalized function $P_{0} f / P_{0} 1$ converges almost everywhere to $f$ on $\partial X$. Many such results are known for $\lambda$-Poisson integrals $P_{\lambda} f$ with $\lambda$ in the positive Weyl chamber. But the case $\lambda=0$ is different, since larger regions of convergence can be used. Some of our results are general, some are given for the bidisk or $\mathrm{SL}(3, \mathbf{R}) / \mathrm{SO}(3)$. The paper extends previous results by the author for the disk and the bidisk.
\end{abstract}

1. Introduction. Let $P=P\left(z, e^{i \theta}\right)$ denote the Poisson kernel in the unit disk $\mathbf{D}$. If $f$ is an $L^{1}$ function on $\mathbf{T}=\partial \mathbf{D}$ and $\lambda \in \mathbf{R}$, the $\lambda$-Poisson integral of $f$ is

$$
P_{\lambda} f(z)=\int_{\mathbf{T}} P\left(z, e^{i \theta}\right)^{\lambda+1 / 2} f\left(e^{i \theta}\right) d \theta .
$$

It is an eigenfunction of the hyperbolic Laplacian in $\mathbf{D}$, with eigenvalue $4 \lambda^{2}-1$. The corresponding normalized $\lambda$-Poisson integral is $\mathscr{P}_{\lambda} f=$ $P_{\lambda} f / P_{\lambda} 1$, where 1 is the constant function on $\mathbf{T}$. Assume $\lambda \geq 0$. Then $\mathscr{P}_{\lambda} f(z) \rightarrow f\left(e^{\imath \theta}\right)$ as $z \rightarrow e^{i \theta}$, uniformly for continuous $f$. This is not true when $\lambda<0$.

Let $f \in L^{1}$. For $\lambda>0$ it is known that $\mathscr{P}_{\lambda} f$ tends nontangentially to $f$ a.e. in $\mathbf{T}$. This means that one lets $z \rightarrow e^{i \theta}$ satisfying a condition $|\arg z-\theta|<$ const. $(1-|z|)$. But when $\lambda=0$, more is true. In fact, $\mathscr{P}_{0} f(z) \rightarrow f\left(e^{i \theta}\right)$ as $z \rightarrow e^{i \theta},|\arg z-\theta|<$ const. $(1-|z|) \log (1-|z|)^{-1}$ for a.a. $\theta$, see Sjögren [16]. We call this weakly tangential convergence; it is false for $\mathscr{P}_{\lambda} f, \lambda>0$. J. Taylor has found a simple description of it in terms of the hyperbolic metric.

In this paper, we shall give some convergence results for $\mathscr{P}_{0} f$ in symmetric spaces, most of which are not valid for other $\mathscr{P}_{\lambda} f$. For rank 1 spaces, the generalization of weakly tangential convergence is rather straightforward, see Theorem 5.4 below. A metric description of the approach region appearing here is also given. Korányi and Picardello [11] 
have an analogous result for homogeneous trees, which in many respects behave like rank 1 symmetric spaces.

For the bidisk $\mathbf{D}^{2}$, Sjögren [14] contains a result of this kind, which we now recall. Poisson integrals $P_{\lambda} f$ and $\mathscr{P}_{\lambda} f, f \in L^{1}\left(\mathbf{T}^{2}\right)$, are here defined as in $\mathbf{D}$, by means of the kernel

$$
P\left(z_{1}, e^{i \theta_{1}}\right)^{\lambda+1 / 2} P\left(z_{2}, e^{i \theta_{2}}\right)^{\lambda+1 / 2}, \quad\left(z_{1}, z_{2}\right) \in \mathbf{D}^{2}, \quad\left(e^{i \theta_{1}}, e^{i \theta_{2}}\right) \in \mathbf{T}^{2} .
$$

In $\mathbf{D}^{2}$ one defines restricted (nontangential) convergence by letting $z=$ $\left(z_{1}, z_{2}\right) \rightarrow\left(e^{i \theta_{1}}, e^{i \theta_{2}}\right) \in \mathbf{T}^{2}$ in such a way that each $z_{j}$ tends nontangentially to $e^{i \theta_{J}}$ and

$$
1-\left|z_{1}\right| \sim 1-\left|z_{2}\right|
$$

Here and in the sequel, $F \sim G$ means $C^{-1} \leq F / G \leq C$, and $C$ denotes many different (large) constants. As is well known, restricted nontangential convergence holds a.e. when $f \in L^{1}$ for the standard Poisson integral $P_{1 / 2} f$, and also for $\mathscr{P}_{\lambda} f, \lambda>0$. Theorem 2 of [14] says that for $\mathscr{P}_{0} f$ one can replace (1.1) by the weaker condition

$$
\log \left(1-\left|z_{1}\right|\right) \sim \log \left(1-\left|z_{2}\right|\right) \text {. }
$$

This is called weakly restricted (nontangential) convergence.

Another natural generalization to $\mathbf{D}^{2}$ of the result in $\mathbf{D}$ would be to use weakly tangential convergence in each $z_{j}$. Then we again have a.e. convergence of $\mathscr{P}_{0} f, f \in L^{1}$, provided we use the restriction condition (1.1). This is Theorem 4.1 below. It is easy to see that (1.1) cannot be replaced by (1.2) here. As in $\mathbf{D}$, one would like to describe Theorem 4.1 in terms of the bihyperbolic distance. However, the natural attempt to do this fails, as verified in $\$ 4$.

In $\$ 5$, we consider a general Riemannian symmetric space $X=G / K$ of the noncompact type. It has a boundary $K / M$. The (standard) notation used here will be explained below. Several modes of convergence at $K / M$ are used, which we briefly recall. See further Korányi [10]. Admissible convergence corresponds to approaching a boundary point $k M$ by means of points $k \exp (H) \cdot x$, with $H \rightarrow+\infty$ in a and $x$ staying in a compact subset of $X$. Letting instead $H \rightarrow+\infty$ along the ray $\left\{t H_{0}: t>0\right\}$ for any fixed $H_{0}$ in the positive Weyl chamber $a_{+}$, one defines restricted convergence. Weakly restricted convergence is defined by letting $H \rightarrow+\infty$ staying in a strict subcone of $\mathfrak{a}_{+}$. This corresponds to (1.2) in $\mathbf{D}^{2}$. In Theorem 5.1, we show that the normalized 0-Poisson integral $\mathscr{P}_{0} f$ converges weakly restrictedly a.e. to $f$ for $f \in L^{1}(K / M)$. When $f$ is continuous, we obtain admissible convergence for $f \in L^{1}(K / M)$, uniformly in $K / M$, which has also been proved by van den Ban and Schlichtkrull [2]. 
Theorem 5.1 was known previously only for rank $X=1$, see Michelson [12]. The rank 1 result Theorem 5.4 was mentioned above.

The last two sections of this paper deal with the rank 2 space $\mathrm{SL}(3, \mathbf{R}) / \mathrm{SO}(3)$. Theorem 6.1 gives a.e. admissible convergence of $\mathscr{P}_{0} f$ for $f \in L^{p}, p>1$. The proof of admissible convergence of $\mathscr{P}_{\lambda} f, \lambda \in \mathfrak{a}_{+}$, in a general symmetric space from Sjögren [17] does not apply to $\mathscr{P}_{0} f$, and we use a method which is specific to $\operatorname{SL}(3, \mathbf{R}) / \mathrm{SO}(3)$.

Finally, Theorem 7.1 is an $\operatorname{SL}(3, \mathbf{R}) / \mathrm{SO}(3)$ analog of the weakly tangential convergence result in the bidisk. With the $\bar{N}$ realization of the boundary, we approach $n_{1} \in \bar{N}$ by means of points

$$
n_{1} \exp \left(t H_{0}\right) n \cdot x, \quad t \rightarrow+\infty .
$$

Here $H_{0} \in a_{+}$is fixed and $x \in X$ stays in a compact set. Further, $n$ satisfies a size condition $|n| \leq C t^{q}$, where $q=q\left(H_{0}\right)$ is positive, which makes this convergence stronger than restricted convergence. We show that $\mathscr{P}_{0} f$ converges to $f$ a.e. in this sense, when $f \in L^{1}$. The proof is rather calculatory and relies on the explicit expression for the Poisson kernel. As a byproduct of the proof, we obtain a restricted convergence result for $\mathscr{P}_{\lambda} f$ with $\lambda \neq 0$ on the boundary of $a_{+}$.

The preparatory $\S 2$ describes the necessary symmetric space theory and notation. Section 3 contains our main lemma and also an estimate for the spherical function corresponding to $\lambda=0$ previously obtained by van den Ban.

The author has profited from a valuable conversation with T. Lyons.

2. Preliminaries about symmetric spaces. Let $X$ be a Riemannian symmetric space of the noncompact type, written as $X=G / K$ in the standard way. The action of $G$ on $X$ is written $g \cdot x$, and $o=e K \in X$. Take an Iwasawa decomposition $K A N$. Thus any $g \in G$ can be written $g=k(g) \exp (H(g)) n(g)$, with $H(g)$ in the Lie algebra a of $A$. As usual, the nilpotent group $\bar{N}$ is the image of $N$ under the Cartan involution, and $M$ is the centralizer of $A$ in $K$.

The (maximal distinguished) boundary of $X$ is $K / M$, and $n \rightarrow k(n) M$ gives a diffeomorphism of $\bar{N}$ onto almost all of $K / M$. Here we refer to the normalized invariant measure $d k M$ in $K / M$. Let $a_{+} \subset \mathfrak{a}$ be the positive Weyl chamber. By means of the Killing form $\langle\cdot, \cdot\rangle$, we identify a with its dual. Then the (restricted) roots are in $a$, and $\rho \in a_{+}$denotes the half-sum of the positive roots, counted with multiplicity. Let $\Sigma^{+}$be the set of positive roots and $\Sigma_{0}$ the set of roots $\alpha$ for which $\alpha / 2$ is not a root. Put $\Sigma_{0}^{+}=\Sigma^{+} \cap \Sigma_{0}$. The Weyl group $W$ consists of linear orthogonal maps 
$a \rightarrow a$ which permute $\Sigma_{0}$. For $s \in W$, we write

$$
(-1)^{s}=(-1)^{\operatorname{card}\left(s\left(\Sigma_{0}^{+}\right) \backslash \Sigma_{0}^{+}\right)}
$$

This expression has the same value for $s$ and $s^{-1}$.

Let $H$ vary in $\mathfrak{a}$. We shall say that $H \rightarrow+\infty$ in $\mathfrak{a}$ if $\langle\alpha, H\rangle \rightarrow+\infty$ for all $\alpha \in \Sigma^{+}$, and similarly we speak of large $H$ in $a$ or $a_{+}$. A function $\varphi: a \rightarrow \mathbf{R}$ is called increasing if $H-H^{\prime} \in \mathfrak{a}_{+}$implies $\varphi(H) \geq \varphi\left(H^{\prime}\right)$. If $H \in \mathfrak{a}$ and $n \in \bar{N}$, we write $n^{H}$ for $\exp (H) n \exp (-H)$. In terms of canonical coordinates in $\bar{N}$, (see [17], Sec. 5), the map $n \rightarrow n^{H}$ is an anisotropic dilation $\left(x_{j}\right) \rightarrow\left(e^{-\left\langle\alpha_{j}, H\right\rangle} x_{\jmath}\right)$, where $\alpha_{j} \in \Sigma^{+}$. The same applies to $N$, except that there is no minus sign in front of $\left\langle\alpha_{\jmath}, H\right\rangle$.

For $\lambda \in \mathfrak{a}$, the $\lambda$-Poisson integral of $f \in L^{1}(K / M)$ is

$$
P_{\lambda} f(g)=P_{\lambda} f(g \cdot o)=\int_{K / M} f(k M) e^{-\left\langle\lambda+\rho, H\left(g^{-1} k\right)\right\rangle} d k M, \quad g \in G .
$$

Considered as a function in $X$, it is a joint eigenfunction of all $G$-invariant differential operators in $X$. Since the Laplacian of $X$ is $G$-invariant, we have Harnack's inequality: $P_{\lambda} f(x) \sim P_{\lambda} f\left(x^{\prime}\right)$ if $x$ and $x^{\prime}$ stay in a compact set in $X$, for $f \geq 0$. By $G$-invariance similarly

$$
P_{\lambda} f(g \cdot x) \sim P_{\lambda} f\left(g \cdot x^{\prime}\right),
$$

uniformly for $g \in G$.

When $f \equiv 1$, we get the spherical function $P_{\lambda} 1$, which is biinvariant, i.e., defined on $K \backslash G / K$. The normalized $\lambda$-Poisson integral of $f$ is $\mathscr{P}_{\lambda} f=P_{\lambda} f / P_{\lambda} 1$. In the case $\lambda=0$, we set for $H \in \mathfrak{a}$

$$
\psi(H)=e^{\langle\rho, H\rangle} P_{0} 1(\exp H) .
$$

This function grows polynomially for large $H$.

The integral defining $P_{\lambda} f$ can be transformed to $\bar{N}$. For $g \cdot o=n_{1} a_{1}$ $\cdot o, n_{1} \in \bar{N}, a_{1} \in A$, this gives

$$
P_{\lambda} f\left(n_{1} a_{1}\right)=\int_{\bar{N}} f(k(n) M) e^{\langle\lambda-\rho, H(n)\rangle} e^{-\left\langle\lambda+\rho, H\left(a_{1}^{-1} n_{1}^{-1} n\right)\right\rangle} d n .
$$

Of course, $d n$ is Haar measure in $\bar{N}$. We often replace $f(k(n) M)$ by $f(n)$ here, working with functions in $\bar{N}$.

Let $a_{1}=\exp H, H \in \mathfrak{a}_{+}$. To estimate $P_{0} 1\left(n_{1} \exp H\right)$, we write $n_{1}=$ $k a n \in K A N$. If $n_{1}$ stays in a compact subset of $\bar{N}$, also $k, a$, and $n$ must stay in compact sets. Then $n_{1} \exp H=k \exp (H) a n^{-H}$, and $n^{-H}$ stays bounded in $N$ for large $H$. Since $P_{0} 1$ is biinvariant, (2.1) gives

$$
\begin{aligned}
P_{0} 1\left(n_{1} \exp H\right) & =P_{0} 1\left(\exp (H) a n^{-H}\right) \sim P_{0} 1(\exp H) \\
& =\psi(H) e^{-\langle\rho, H\rangle} .
\end{aligned}
$$


The Poisson kernel in $\bar{N}$ is defined by

$$
P(n)=e^{-2\langle\rho, H(n)\rangle} .
$$

For any function $F$ in $\bar{N}$, we write $\tilde{F}(n)=F\left(n^{-1}\right)$. Also, the normalized contraction of $F$ is defined by $F_{H}(n)=e^{2\langle\rho, H\rangle} F\left(n^{-H}\right)$ for $H \in \mathfrak{a}$. Then $\int F_{H} d n=\int F d n$ if $F \in L^{1}$. If $f \geq 0$ has compact support in $\bar{N}$, and $n_{1}$ stays in a compact set, it follows from (2.2) and (2.3) that

$$
\begin{aligned}
\mathscr{P}_{0} f\left(n_{1} \exp H\right) & \leq C \psi(H)^{-1} e^{2\langle\rho, H\rangle} \int_{\bar{N}} f(n) e^{-\left\langle\rho, H\left(\left(n_{1}^{-1} n\right)^{-H}\right)\right\rangle} d n \\
& =C \psi(H)^{-1} f * \tilde{P}_{H}^{1 / 2}\left(n_{1}\right) .
\end{aligned}
$$

Here $\tilde{P}_{H}^{1 / 2}$ means $(\sqrt{\tilde{P}})_{H}$.

From Knapp and Williamson [9], Prop. 5.1, we know that $1 / P$ is a finite sum of nonnegative homogeneous polynomials. This means that

$$
1 / P\left(n^{-H}\right)=\sum_{0}^{\nu_{0}} e^{2\left\langle\beta_{\nu}, H\right\rangle} Q_{\nu}(n),
$$

and each $Q_{\nu}$ is a nonnegative polynomial function of the canonical coordinates of $n$. Further, $Q_{0} \equiv 1$. The $\beta_{\nu}$ are linear combinations of positive roots with nonnegative integer coefficients, and only $\beta_{0}$ is 0 . Moreover, $\left\langle 2 \rho-\beta_{\nu}, H\right\rangle \geq 0$ when $H \in \mathfrak{a}_{+}$, for all $\nu$.

Given a fixed vector $H_{0} \in \mathfrak{a}_{+}$, we can find a smooth homogeneous gauge $|\cdot|$ in $\bar{N}$. This is a function $\bar{N} \rightarrow\{t \geq 0\}$ which is smooth outside $e$ and vanishes only at $e$. It also satisfies $\left|n^{-1}\right|=|n|$ and

$$
\left|n^{t H_{0}}\right|=e^{-t}|n|, \quad t \in \mathbf{R} \text {. }
$$

This implies the quasinorm property

$$
\left|n n^{\prime}\right| \leq C\left(|n|+\left|n^{\prime}\right|\right) \text {. }
$$

The ball $\{n:|n| \leq r\}$ is denoted by $B(r)$. Its Haar measure $|B(r)|$ is proportional to $r^{2\left\langle\rho, H_{0}\right\rangle}$.

The gauge will be related to the canonical coordinates of $n$ by

$$
C^{-1} \max \left|x_{j}\right|^{\alpha} \leq|n| \leq C \max \left|x_{j}\right|^{\beta}
$$

for some $C$ and $\alpha, \beta>0$.

Finally, we write $e_{k}=e^{-2^{k}}, k=0,1, \ldots$, and it will be convenient to let $e_{k}$ for $k<0$ denote large constants depending on the context.

3. Auxiliary results. We first give an $\bar{N}$ version of the method of $k$-pieces from Sjögren [14], cf. in particular Lemma 2. Let $L \subset \bar{N}$ be compact, and extend any function in $L$ by 0 to all of $\bar{N}$. 
Lemma 3.1. (a) Assume the sublinear operators $\left(T_{k}\right)_{1}^{\infty}$ are defined in $L^{1}(L)$, take values which are nonnegative measurable functions in $L$, and satisfy the following for some $C_{1}<\infty$.

(i) Each $T_{k}$ is of weak type $(1,1)$ with constant at most $C_{1}$.

(ii) The value $T_{k} f(n), n \in L$, depends only on the restriction of $f$ to the ball $n B\left(e_{k}\right)$.

(iii) For some natural number $N$,

$$
\left\|T_{k} f\right\|_{L^{\infty}} \leq C_{1} \sup _{n}\left|B\left(e_{k+N}\right)\right|^{-1} \int_{n B\left(e_{k+N}\right)}|f| d n .
$$

Then the operator $f \rightarrow \sup _{k} T_{k} f$ is of weak type $(1,1)$ with constant at most $C C_{1} N, C=C(L)$.

(b) The hypothesis (iii) in (a) can be replaced by the following:

(iii') Each $T_{k}$ is given by

$$
T_{k} f(n)=\sup _{i \in I_{k}}|f| * K_{l}(n),
$$

where $I_{k}$ is an index set and the kernels $K_{l}$ satisfy $\int K_{l}^{*}(n) d n \leq C_{1}$. Here, for $i \in I_{k}$,

$$
K_{l}^{*}(n)=\sup _{n^{\prime} \in B\left(e_{k+N}\right)} K_{i}\left(n n^{\prime}\right) .
$$

Proof of (a). We shall apply Lemma 1 of [14], recalled below. Small values of $k$ will cause minor complications, so we consider only $k \geq A$, where $A$ is a suitable large integer. This is enough, because of (i). For each $k \geq A$, choose in $\bar{N}$ a lattice of cubes which are defined in terms of the canonical coordinates and whose sides are equal and approximately $e_{k-A}$. These cubes are called $k$-pieces. We also choose these lattices in such a way that each $k$-piece is the union of the $(k+1)$-pieces contained in it. (Neglect the boundaries of the cubes.) Moreover, the ratio between the sides of a $k$-piece and a $(k+1)$-piece should be odd.

Lemma 1 of [14] says that the operator $f \rightarrow \sup _{k} T_{k}^{\prime} f$ is of weak type $(1,1)$ provided that

$(\alpha)$ the sublinear operators $T_{k}^{\prime}$ are uniformly of weak type $(1,1)$;

$(\beta)$ the restriction of $T_{k}^{\prime} f$ to a $k$-piece $P$ depends only on $\left.f\right|_{P}$;

$(\gamma)\left\|T_{k}^{\prime} f\right\|_{L^{\infty}} \leq C \sup |P|^{-1} \int_{P}|f| d n$, the supremum taken over all $\left(k+N^{\prime}\right)$-pieces $P$, for some fixed $N^{\prime} \in\{1,2, \ldots\}$. 
From the proof in [14], it is immediately seen that the constant in the conclusion is $O\left(N^{\prime}\right)$.

Our $T_{k}^{\prime}$ will be slight modifications of the $T_{k}$. Because of (2.6), the variation of each coordinate in the ball $B\left(e_{k}\right)$ is at most $e_{k-C}$, which is much smaller than $e_{k-A}$ if $A$ is suitably chosen. The same holds for any ball $n B\left(e_{k}\right), n \in L$. Thus (ii) implies that $T_{k} f(n)$ depends only on the restriction of $f$ to the $k$-piece containing $n$, except when $n$ is very close to the boundary of some $k$-piece. In this last case, we let $T_{k}^{\prime} f(n)=0$, otherwise $T_{k}^{\prime} f(n)=T_{k} f(n)$. Then $(\beta)$ holds, and also $(\alpha)$.

To obtain $(\gamma)$ from (iii), it is enough to estimate the mean of $|f|$ in $n B\left(e_{k+N}\right)$ by $C$ times the largest of the means of $|f|$ in those $\left(k+N^{\prime}\right)$ pieces intersecting $n B\left(e_{k+N}\right)$. For this we need only verify that these $\left(k+N^{\prime}\right)$-pieces have total measure at most $C\left|B\left(e_{k+N}\right)\right|$, or that they are all contained in $n B\left(C e_{k+N}\right)$. But any point $n^{\prime}$ in such a piece is obtained by changing the coordinates of a point $n^{\prime \prime} \in n B\left(e_{k+N}\right)$ by at most $C e_{k+N^{\prime}-A}$. Since $n^{\prime}$ and $n^{\prime \prime}$ stay in a compact set, the coordinates of $\left(n^{\prime \prime}\right)^{-1} n^{\prime}$ are $O\left(e_{k+N^{\prime}-A}\right)$. Then (2.6) implies $\left|\left(n^{\prime \prime}\right)^{-1} n^{\prime}\right| \leq e_{k+N^{\prime}-A-C} \leq$ $e_{k+N}$, if $N^{\prime} \geq N+A+C$. It follows that

$$
n^{\prime}=n^{\prime \prime}\left(n^{\prime \prime}\right)^{-1} n^{\prime} \in n B\left(e_{k+N}\right) B\left(e_{k+N}\right) \subset n B\left(C e_{k+N}\right),
$$

and $(\gamma)$ is verified.

Thus $\sup _{k} T_{k}^{\prime}$ is of weak type $(1,1)$, and the corresponding constant is at most $C N^{\prime} \leq C N$. To extend this to $T_{k}$, we must recover those values of $T_{k} f$ which we lost when defining $T_{k}^{\prime}$. This is done as in the proof of Lemma 2 in [14]. Indeed, one can repeat the argument just given with the lattices translated half the side of an $A$-piece in one or more coordinate directions. Here translation is taken in the Euclidean sense. Since the ratios of the cube sides are odd, this will make us catch all of $T_{k} f$ in $2^{\operatorname{dim} \bar{N}}$ steps. Part (a) is proved.

Proof of (b). Assuming (iii'), we shall prove (iii). One can clearly assume $\operatorname{supp} K_{i} \subset L^{-1} L$. We consider the functions

$$
K_{i}^{* *}(n)=\sup _{\left|n^{\prime}\right| \leq e_{k+N+C}} K_{l}\left(n^{\prime} n\right), \quad i \in I_{k} .
$$

Take $n^{\prime} \in B\left(e_{k+N+C}\right)$ and write $n^{\prime} n=n n^{-1} n^{\prime} n$. The coordinates of $n^{-1} n^{\prime} n$ are polynomials in the coordinates of $n$ and $n^{\prime}$, and vanish for $n^{\prime}=e$. They are thus bounded by const. times the largest coordinate of $n^{\prime}$ when $n$ and $n^{\prime}$ stay bounded. Then $\left|n^{-1} n^{\prime} n\right| \leq e_{k+N}$ because of (2.6), if $C$ is suitably chosen. Hence, $K_{i}^{* *} \leq C K_{l}^{*}$ and $\int K_{i}^{* *} d n \leq C C_{1}$. 
With $i \in I_{k}$ and $f \geq 0$, we have

$$
f * K_{l}\left(n_{1}\right)=\int f(n) K_{l}\left(n^{-1} n_{1}\right) d n \leq \int f(n) K_{i}^{* *}\left(n^{\prime} n^{-1} n_{1}\right) d n,
$$

for any $n^{\prime} \in B\left(e_{k+N+C}\right)$. Thus,

$$
f * K_{l}\left(n_{1}\right) \leq \int f(n) d n\left|B\left(e_{k+N+C}\right)\right|^{-1} \int_{\left|n^{\prime}\right| \leq e_{k+N+C}} K_{\imath}^{* *}\left(n^{\prime} n^{-1} n_{1}\right) d n^{\prime} .
$$

Transforming $n \rightarrow n^{-1} n^{\prime}$, we get

$$
f * K_{l}\left(n_{1}\right) \leq \int K_{i}^{* *}\left(n n_{1}\right) d n\left|B\left(e_{k+N+C}\right)\right|^{-1} \int_{\left|n^{\prime}\right| \leq e_{k+N+C}} f\left(n^{-1} n^{\prime}\right) d n^{\prime} .
$$

Since $K_{l}^{* *} \in L^{1}$, (iii) now follows, with $N+C$ instead of $N$. Lemma 3.1 is proved.

Next, we consider $P(n)^{1 / 2}$ and $\psi(H)$.

LEMMA 3.2. For each $n \in \bar{N}$ the expression

$$
P_{H}^{1 / 2}(n)=e^{2\langle\rho, H\rangle} P\left(n^{-H}\right)^{1 / 2}
$$

is increasing as a function of $H \in \mathfrak{a}$.

This is almost Lemma 3.5 of Sjögren [13]. For the proof, we need only observe that all the terms in the sum in (2.5) become decreasing in $H$ when multiplied by $e^{-4\langle\rho, H\rangle}$.

Via an integration, this lemma implies the known fact that the function $\psi$ is increasing (Harish-Chandra [5], Lemma 36 p. 281). In [1], Theorem 6.6, van den Ban determined its order of magnitude at $+\infty$. We shall do this again, without insisting on the value of the corresponding constant. For the case $X=\operatorname{SL}(3, \mathbf{R}) / \mathrm{SO}(3)$, see also Herz [8]. Let $\pi$ be the polynomial

$$
\pi(H)=\prod_{\alpha \in \Sigma_{0}^{+}}\langle\alpha, H\rangle, \quad H \in \mathfrak{a} .
$$

With any $H \in \mathfrak{a}$, we can associate the differential operator $\partial(H)$ defined in $\mathfrak{a}$ as differentiation along the vector $H$. Then $\partial$ can be extended to a homomorphism, also denoted $\partial$, from the ring of polynomials on $a$ into that of differential operators in $\mathfrak{a}$.

Proposition 3.3. For large $H \in \mathfrak{a}_{+}$, one has $\psi(H) \sim \pi(H)$.

Proof. When $\lambda \in \mathrm{a}_{+}$, one can expand $P_{\lambda} 1$ :

$$
P_{\lambda} 1(\exp H)=\sum_{s \in W} c(s \lambda) e^{\langle s \lambda-\rho, H\rangle} \sum_{\mu \in \Lambda} \Gamma_{\mu}(s \lambda) e^{-\langle\mu, H\rangle},
$$


see Helgason [7], Theorem IV.5.5 p. 430. Here $c$ is Harish-Chandra's $c$-function, and $\Lambda$ is the set of linear combinations of the simple positive roots with nonnegative integer coefficients. The quantities $\Gamma_{\mu}(\lambda)$ are given by $\Gamma_{0}(\lambda)=1$ and by the recursion formula (12) of [7], IV.5 p. 427. To deal with the singularity of $c$ at 0 , we follow Harish-Chandra and write

$$
P_{0} 1(\exp H)=c_{1}\left\{\partial(\pi)\left(\pi(\lambda) P_{\lambda} 1(\exp H)\right)\right\}_{\lambda=0},
$$

where the operator $\partial(\pi)$ is taken with respect to $\lambda$ and $c_{1} \neq 0$ is a constant. See also Exercise IV.B.1, p. 483 of [7]. Then (3.1) gives

$$
\text { (3.2) } \psi(H)=c_{1}\left\{\partial(\pi)\left(\sum_{s \in W} \pi(\lambda) c(s \lambda) e^{\langle s \lambda, H\rangle} \sum_{\mu \in \Lambda} \Gamma_{\mu}(s \lambda) e^{-\langle\mu, H\rangle}\right)\right\}_{\lambda=0} \text {. }
$$

Let $s \in W$. At $\lambda=0$, the function $\pi(\lambda) c(s \lambda)$ is smooth and takes the value $c_{2}(-1)^{s}, c_{2} \neq 0$, as seen from the explicit expression for $c(\lambda)$ (formula (43) of [7], IV.6 p. 447).

In the expansion of the derivative in (3.2), consider those terms obtained by letting $\partial(\pi)$ act only on $e^{\langle s \lambda, H\rangle}$ and taking only $\mu=0$ in the last sum. These terms will sum up to

$$
\sum_{s \in W} c_{2}(-1)^{s} \pi(s H)=c_{2} \pi(H)
$$

We shall see that the remaining part of (3.2) is much smaller, for large $H$.

Let

$$
Q_{\mu}=\sup _{\pi^{\prime}}\left|\left\{\partial\left(\pi^{\prime}\right) \Gamma_{\mu}(\lambda)\right\}_{\lambda=0}\right|,
$$

the supremum taken over $\pi^{\prime}$ of type

$$
\pi^{\prime}(H)=\prod_{\alpha \in \Sigma^{\prime}}\langle\alpha, H\rangle, \quad \Sigma^{\prime} \subset \Sigma_{0}^{+} .
$$

We claim that there exists $C$ such that for all $\mu \in \Lambda$

$$
Q_{\mu} \leq C e^{\langle\mu, \rho\rangle} \text {. }
$$

This is a simple extension of the estimate for $\Gamma_{\mu}$ given in Lemma IV.5.3 of [7], where we have chosen $H=\rho$. The proof is similar: by differentiating the recursion formula for $\Gamma_{\mu}$, one obtains a recursive inequality for $Q_{\mu}$ which is like (14) of [7], IV.5.3. After that, Helgason's arguments can be repeated and give (3.3).

Since now (3.3) allows us to sum in $\mu$, it is not hard to see that the remaining part of (3.2) is $o(\pi(H))$ as $H \rightarrow+\infty$. The proposition follows. 
4. The bidisk. Whenever convenient, we consider $\mathbf{T}$ as $\mathbf{R} / 2 \pi \mathbf{Z}$ rather than $\{|z|=1\}$. Define for $\left(\theta_{1}, \theta_{2}\right) \in \mathbf{T}^{2}$ the region of restricted weakly tangential convergence by

$$
\begin{aligned}
& \Omega_{\theta_{1}, \theta_{2}}=\left\{\left(z_{1}, z_{2}\right)\right.=\left(\left(1-t_{1}\right) e^{i \varphi_{1}},\left(1-t_{2}\right) e^{i \varphi_{2}}\right) \in \mathbf{D}^{2}: \\
&\left.0<t_{1} \sim t_{2}<1 / 2,\left|\varphi_{i}-\theta_{i}\right| \leq C t_{i} \log t_{i}^{-1}, i=1,2\right\} .
\end{aligned}
$$

TheOREM 4.1. Let $f \in L^{1}\left(\mathbf{T}^{2}\right)$. Then for a.a. $\left(\theta_{1}, \theta_{2}\right) \in \mathbf{T}^{2}$

$$
\mathscr{P}_{0} f\left(z_{1}, z_{2}\right) \rightarrow f\left(\boldsymbol{\theta}_{1}, \boldsymbol{\theta}_{2}\right)
$$

as $\left(z_{1}, z_{2}\right)$ tends to $\left(e^{i \theta_{1}}, e^{i \theta_{2}}\right)$ staying in $\Omega_{\theta_{1}, \theta_{2}}$.

Proof. In the disk, the normalizing factor $P_{0} 1$ behaves like $(1-|z|)^{1 / 2} \log (1-|z|)^{-1}$, and in $\mathbf{D}^{2}$ like a tensor product of two such factors. Therefore, $\mathscr{P}_{0} f\left(z_{1}, z_{2}\right)$ is given by a convolution in $\mathbf{T}^{2}$ of $f$ and a kernel which is

$$
\sim \frac{1}{\log t_{1} \log t_{2}} \frac{1}{t_{1}+\left|\theta_{1}\right|} \frac{1}{t_{2}+\left|\theta_{2}\right|}, \quad t_{i}=1-\left|z_{i}\right| .
$$

Define in $\mathbf{T}^{2}$

$$
K_{t}\left(\theta_{1}, \theta_{2}\right)=\frac{1}{(\log t)^{2}} \frac{1}{t+\left(\left|\theta_{1}\right|-t \log t^{-1}\right)_{+}} \frac{1}{t+\left(\left|\theta_{2}\right|-t \log t^{-1}\right)_{+}} .
$$

If for simplicity we take $C=1$ in the definition of $\Omega_{\theta_{1}, \theta_{2}}$, it follows that

$$
\sup _{\Omega_{\theta_{1}, \theta_{2}}}\left|\mathscr{P}_{0} f\right| \leq C \sup _{0<t<1 / 2}|f| * K_{t}\left(\theta_{1}, \theta_{2}\right) .
$$

Since convergence holds for continuous $f$, we need only deduce a weak type $(1,1)$ estimate for the operator defined by the left-hand side here.

To do this, we divide $K_{t}\left(\theta_{1}, \theta_{2}\right)$ into four parts. That part where $\left|\theta_{1}\right|,\left|\theta_{2}\right| \leq 2 t \log t^{-1}$ leads to the mean value of $|f|$ in squares of side $4 t \log t^{-1}$, which is easy to control. For $\left|\theta_{1}\right|,\left|\theta_{2}\right|>2 t \log t^{-1}$, we can estimate the kernel by

$$
C \frac{1}{(\log t)^{2}} \frac{1}{t+\left|\theta_{1}\right|} \frac{1}{t+\left|\theta_{2}\right|}
$$

This corresponds to restricted nontangential convergence and can be controlled, see Theorem 4 of [14] or Theorem 5.1 below.

The third part of $K_{t}$ is defined by $\left|\theta_{1}\right| \leq 2 t \log t^{-1}<\left|\theta_{2}\right|$. (The fourth part is analogous and not discussed.) We estimate the supremum in $t$ of 
the convolution of $|f|$ with the kernel

$$
\frac{1}{t \log t^{-1}} x_{\left|\theta_{1}\right| \leq 2 t \log t^{-1}} \frac{1}{\log t^{-1}} \frac{1}{t \log t^{-1}+\left|\theta_{2}\right|} .
$$

Replacing $2 t \log t^{-1}$ by $t$, we obtain the kernel

$$
L_{t}\left(\theta_{1}, \theta_{2}\right)=t^{-1} \chi_{\left|\theta_{1}\right| \leq t} \frac{1}{\log t^{-1}} \frac{1}{t+\left|\theta_{2}\right|} .
$$

Assume $e_{k} \leq t \leq e_{k-1}$. If $e_{k-\nu} \leq\left|\theta_{2}\right| \leq e_{k-\nu-1}$ with $\nu \in\{0,1, \ldots, k\}$, the last factor in (4.2) is of course dominated by $C\left(e_{k-\nu}+\left|\theta_{2}\right|\right)^{-1}$. This gives

$$
L_{t}\left(\theta_{1}, \theta_{2}\right) \leq C t^{-1} \chi_{\left|\theta_{1}\right| \leq t} \sum_{\nu=0}^{k} \frac{2^{-k}}{e_{k-\nu}+\left|\theta_{2}\right|} \chi_{\left|\theta_{2}\right| \leq e_{k-\nu-1}} .
$$

Here it is important that those factors which contain $\theta_{2}$ do not depend on $t$. We must estimate

(4.4) $\sum_{\nu=0}^{\infty} 2^{-\nu} \sup _{k \geq \nu} \sup _{e_{k} \leq t \leq e_{k-1}}|f| *\left(t^{-1} \chi_{\left|\theta_{1}\right| \leq t} \frac{2^{\nu-k}}{e_{k-\nu}+\left|\theta_{2}\right|} \chi_{\left|\theta_{2}\right| \leq e_{k-\nu-1}}\right)$.

For each $\nu$, we apply Lemma 2 of [14] to the supremum in $k$ here.

To verify the hypotheses of this lemma, we estimate the supremum in $t$ in (4.4). By integrating first in $\theta_{2}$ and then in $\theta_{1}$, we see that this supremum is essentially the standard maximal function taken in $\theta_{1}$ of a convolution in $\theta_{2}$ of $f$ and a function whose $L^{1}$ norm is bounded. Therefore, this supremum defines an operator of weak type $(1,1)$, uniformly in $k$. Further, it depends only on the values of $f$ in a rectangle of sides $e_{k-1}$ and $e_{k-\nu-1}$, centered at the point considered. Finally, we have a stability condition like (iii') of our Lemma 3.1, where we can move at most $e_{k}$ and $e_{k-\nu}$ in the first and second variables, respectively, when defining the starred kernels.

Hence, Lemma 2 of [14] applies, with $N=1$. This means that each term of (4.4) gives a weak type $(1,1)$ operator with a constant which is $O\left(2^{-\nu}\right)$. We can then sum in weak $L^{1}$ (cf. Stein [18], Sec. 6) and obtain an operator of weak type $(1,1)$. This takes care of the third part of our kernel and completes the proof of Theorem 4.1.

Let $d$ denote the hyperbolic distance in $\mathbf{D}$, normalized by $d s=$ $\left(1-|z|^{2}\right)^{-1}|d z|$, and $D$ the corresponding bihyperbolic distance in $\mathbf{D}^{2}$. As shown by J. Taylor (unpublished), the convergence condition for $\mathscr{P}_{0} f$ in D,

$$
|\arg z-\theta| \leq C(1-|z|) \log (1-|z|)^{-1}
$$


is equivalent to

$$
d\left(z, r_{\theta}\right) \leq \frac{1}{2} \log d(z, 0)+C,
$$

up to the values of $C$. Here $r_{\theta}$ is the geodesic ray $\left\{r e^{i \theta}: 0 \leq r<1\right\}$, and we similarly let $R_{\theta_{1}, \theta_{2}}=\left\{\left(r e^{i \theta_{1}}, r e^{i \theta_{2}}\right): 0 \leq r<1\right\} \subset \mathbf{D}^{2}$. Inequality (4.6) should of course be compared to the condition $d\left(z, r_{\theta}\right) \leq C$, which defines nontangential convergence in $\mathbf{D}$. In $\mathbf{D}^{2}$, restricted nontangential convergence is similarly described by $D\left(\left(z_{1}, z_{2}\right), R_{\theta_{1}, \theta_{2}}\right) \leq C$.

A natural question is now whether the convergence condition of Theorem 4.1 can be similarly described in terms of $D$. We shall prove below that any domain $W_{\theta_{1}, \theta_{2}}$ containing $\Omega_{\theta_{1}, \theta_{2}}$ which one can define by means of an inequality $D\left(z, R_{\theta_{1}, \theta_{2}}\right) \leq \psi(D(z, 0)), z \in \mathbf{D}^{2}$, for some function $\psi$, will be too large to allow a.e. convergence of $\mathscr{P}_{0} f, f \in L^{1}$. Here it is assumed that $W_{\theta_{1}, \theta_{2}}$ is obtained by rotating $W=W_{0,0}$, which we consider first.

If $s_{0}$ is large and $\theta=s_{0} e^{-2 s_{0}}$, the point $z=\left(e^{i \theta} \tanh s_{0}, e^{i \theta} \tanh s_{0}\right)$ belongs to $\Omega_{0,0}$. Clearly $D(z, 0)=s_{0} \sqrt{2}$. We shall estimate the distance $D_{1}$ from $z$ to $R_{0,0}$ and start by observing that $D_{1}=d_{1} \sqrt{2}$, where $d_{1}=$ $d\left(e^{\imath \theta} \tanh s_{0}, r_{0}\right)$.

To estimate $d_{1}$, we let $\gamma:\left[0, d_{1}\right] \rightarrow \mathbf{D}$ be the shortest geodesic arc from $e^{\imath \theta} \tanh s_{0}$ to $r_{0}$, parametrized by arc length. Then $\gamma$ is a Euclidean circle arc, orthogonal to $r_{0}$ at $\gamma\left(d_{1}\right)=\tanh s$, and one has $s<s_{0}$. Let $\gamma\left(t_{0}\right)$ be the Euclidean midpoint of $\gamma$. Then $\arg \gamma\left(t_{0}\right)>\theta / 2$, and $1-\left|\gamma\left(t_{0}\right)\right|>$ $\left(1-\gamma\left(d_{1}\right)\right) / 2 \sim e^{-2 s}$. Considering the variation of $|\gamma(t)|$ in $\left[0, t_{0}\right]$ and that of $\arg \gamma(t)$ in $\left[t_{0}, d_{1}\right]$, we find that the length $d_{1}$ is at least $s_{0}-s-C$ $+\theta / 2 \cosh ^{2} s$. Now insert the value of $\theta$ and minimize with respect to $s$. We find $d_{1} \geq \frac{1}{2} \log s_{0}-C$ and thus

$$
D_{1} \geq \frac{1}{\sqrt{2}} \log s_{0}-C=\frac{1}{\sqrt{2}} \log D(z, 0)-C .
$$

Incidentally, this estimate for $d_{1}$ makes it easy to verify the equivalence of (4.5) and (4.6). This will be done in greater generality in the proof of Theorem 5.4.

This means that any candidate for $W$ must contain the region

$$
W=\left\{z \in \mathbf{D}^{2}: D(z, 0) \text { large and } D\left(z, R_{0,0}\right) \leq(1 / \sqrt{2}) \log D(z, 0)-C\right\} \text {. }
$$

We need an observation about this $W$. If $s_{1}=s+\sqrt{2}^{-1} \log s-C_{1}$ and $C_{1}$ is large enough, the point $z=\left(\tanh s, e^{i \theta} \tanh s_{1}\right)$ belongs to $W$ for $|\theta| \leq e^{-2 s}$ and large $s$. This is seen by measuring a path from $z$ to $R_{0,0}$ defined as follows. Keep the first coordinate constant at $\tanh s$ and move the second coordinate first radially from $e^{i \theta} \tanh s_{1}$ to $e^{i \theta} \tanh s$ and then 
along the circle $\left|z_{2}\right|=\tanh s$ to tanh $s$. One finds $D\left(z, R_{0,0}\right) \leq s_{1}-s+C$. Since $D(z, 0)=s \sqrt{2}+O(\log s)$, it follows that $z \in W$.

To disprove a.e. convergence of $\mathscr{P}_{0} f, f \in L^{1}\left(T^{2}\right)$, as $z$ tends to $\left(e^{i \theta_{1}}, e^{i \theta_{2}}\right)$ staying in $W_{\theta_{1}, \theta_{2}}$, we replace $f$ by a point mass $\delta$ at $(0,0) \in \mathbf{T}^{2}$. Let $\theta_{1}$ and $\theta_{2}$ be small and positive, choose $s$ so that $e^{-2 s}=\theta_{2}$, and let $s_{1}$ be as before. Then $z=\left(e^{i \theta_{1}} \tanh s, \tanh s_{1}\right) \in W_{\theta_{1}, \theta_{2}}$ by the above, and

$$
\mathscr{P}_{0} \delta(z) \geq \frac{1}{C s^{2}} \frac{1}{e^{-2 s}+\left|\theta_{1}\right|} \frac{1}{e^{-2 s_{1}}} .
$$

For $\theta_{2}<\theta_{1}$ we get

$$
\sup _{W_{\theta_{1}, \theta_{2}}} \mathscr{P}_{0} \delta \geq \frac{1}{C\left|\log \theta_{2}\right|^{2-\sqrt{2}}} \frac{1}{\theta_{1} \theta_{2}} .
$$

This is easily seen to disprove a weak type $(1,1)$ estimate for the maximal function corresponding to $W$. From general theorems, it now follows that a.e. convergence does not hold for all $L^{1}$ functions, see Chapter 2 of de Guzmán [4].

5. Results for general symmetric spaces. Take $X$ as in $\S 2$, and let the gauge in $\bar{N}$ be defined with $H_{0}=\rho$. We call $V \subset \mathfrak{a}_{+}$a strict subcone of $a_{+}$if $V$ is a cone, i.e., $\mathbf{R}_{+} V=V$, and the closure of $V$ is contained in $a_{+} \cup\{0\}$.

TheOREM 5.1. Let $f \in L^{1}(K / M)$ and assume that $D \subset X$ is compact and $V$ is a strict subcone of $\mathfrak{a}_{+}$. Then

$$
\mathscr{P}_{0} f(k \exp (H) \cdot x) \rightarrow f(k M) \text { as } H \rightarrow+\infty, H \in V,
$$

uniformly for $x \in D$, for a.a. $k M \in K / M$. If $f$ is continuous, one has admissible convergence, i.e., the condition $H \in V$ can be deleted, uniformly in $K / M$.

The result for continuous $f$ here is also in van den Ban and Schlichtkrull [2, Cor. 16.6]. Before proving the theorem, we give a lemma about the behavior of $P(n)$ for large $n$.

LEMMA 5.2. Given $\delta>0$, there exists a constant $B<\infty$ such that

$$
P(n) \leq \delta P\left(n^{H}\right)
$$

for $|n|>B$ and large $H \in \mathfrak{a}_{+}$. 
Proof. Because of (2.5), we must prove

$$
\sum_{\nu=0}^{\nu_{0}} e^{-2\left\langle\beta_{\nu}, H\right\rangle} Q_{\nu}(n) \leq \delta \sum_{\nu=0}^{\nu_{0}} Q_{\nu}(n) .
$$

For $\nu>0$, we have termwise inequalities when $H$ is large, since $\beta_{\nu} \neq 0$. Term number 0 in the left-hand side is 1 , and it remains to see that $1 \leq \delta / P(n)$. But this holds for large $n$, see [9], Prop. 5.5. The lemma follows.

Proof of Theorem 5.1. Consider first continuous $f$ in $K / M$. We must show that the operators which map $f$ to the functions

$$
k M \rightarrow \mathscr{P}_{0} f(k \exp (H) \cdot x)
$$

form an approximate identity in $K / M$ as $H \rightarrow+\infty, x \in D$. Since the corresponding kernels are positive and constant functions are preserved, it is enough to prove convergence at $e M \in K / M$ for $f \geq 0$ vanishing near $e M$. Harnack's inequality (2.1) allows us to take $D=\{o\}$. Thus we must verify that $\mathscr{P}_{0} f(\exp H) \rightarrow 0$ as $H \rightarrow+\infty$. Because of (2.2) and the definition of $\psi$, this holds if

(5.1) $\psi(H)^{-1} e^{2\langle\rho, H\rangle} \int_{|n|>\varepsilon} e^{-\langle\rho, H(n)\rangle} e^{-\left\langle\rho, H\left(n^{-H}\right)\right\rangle} d n \rightarrow 0 \quad$ as $H \rightarrow+\infty$ for any $\varepsilon>0$.

Let $\delta>0$ and take $B$ as in Lemma 5.2. Write the integral in (5.1) as $\int_{|n|>B}+\int_{\varepsilon<|n|<B}$. In the first term here, we apply Lemma 5.2 and then transform $n \rightarrow n^{-H}$. This gives

$$
\begin{aligned}
\psi(H)^{-1} e^{2\langle\rho, H\rangle} \int_{|n|>B} e^{-\langle\rho, H(n)\rangle} e^{-\left\langle\rho, H\left(n^{-H}\right)\right\rangle} d n \\
\quad \leq \sqrt{\delta} \psi(H)^{-1} e^{2\langle\rho, H\rangle} \int_{|n|>B} e^{-\left\langle\rho, H\left(n^{H}\right)\right\rangle} e^{-\left\langle\rho, H\left(n^{-H}\right)\right\rangle} d n \\
\quad \leq \sqrt{\delta} \psi(H)^{-1} e^{4\langle\rho, H\rangle} \int e^{-\langle\rho, H(n)\rangle} e^{-\left\langle\rho, H\left(n^{-2 H}\right)\right\rangle} d n \\
\leq \sqrt{\delta} \psi(2 H) / \psi(H) \leq C \sqrt{\delta},
\end{aligned}
$$

where the last inequality holds for large $H$ because of Proposition 3.3.

For the integral over $R=\{n: \varepsilon<|n|<B\}$, we first introduce the regions $R_{m}=\left\{n^{m \delta H}: n \in R\right\}, m=1,2, \ldots$ If $H$ is large, the $R_{m}$ will be pairwise disjoint and contained in $B(1)$. Estimating $e^{-\langle\rho, H(n)\rangle}$ by 1 and 
transforming $n \rightarrow n^{-m \delta H}$, we get

$$
\begin{aligned}
& \psi(H)^{-1} e^{2\langle\rho, H\rangle} \int_{R} e^{-\langle\rho, H(n)\rangle} e^{-\left\langle\rho, H\left(n^{-H}\right)\right\rangle} d n \\
& \quad \leq \psi(H)^{-1} e^{2(1+m \delta)\langle\rho, H\rangle} \int_{R_{m}} e^{-\left\langle\rho, H\left(n^{-(1+m \delta) H}\right)\right\rangle} d n .
\end{aligned}
$$

For $1 \leq m \leq\left[\delta^{-1}\right]$, Lemma 3.2 implies that this is at most

$$
\psi(H)^{-1} e^{4\langle\rho, H\rangle} \int_{R_{m}} e^{-\left\langle\rho, H\left(n^{-2 H}\right)\right\rangle} d n .
$$

In this integral, we insert a factor $e^{-\langle\rho, H(n)\rangle}$, which is bounded below in $B(1)$. Then we sum in $m$, getting

$$
\begin{aligned}
{\left[\delta^{-1}\right] \psi(H)^{-1} e^{2\langle\rho, H\rangle} } & \int_{\varepsilon}\langle|n|\langle B \\
& e^{-\langle\rho, H(n)\rangle} e^{-\left\langle\rho, H\left(n^{-H}\right)\right\rangle} d n \\
& \leq C \psi(H)^{-1} e^{4\langle\rho, H\rangle} \int_{B(1)} e^{-\langle\rho, H(n)\rangle} e^{-\left\langle\rho, H\left(n^{-2 H}\right)\right\rangle} d n \\
& \leq C \psi(2 H) / \psi(H) \leq C .
\end{aligned}
$$

Dividing by $\left[\delta^{-1}\right]$, we obtain an inequality which together with (5.2) implies (5.1). This proves the last statement in Theorem 5.1.

Now consider $f \in L^{1}(K / M)$. The method of [15], $\$ 3$ p. 49, shows that it is enough to take $f \in L^{1}(\bar{N})$ with support contained in a compact set $L \subset \bar{N}$, and prove a.e. convergence in $L$ only. Modifying the gauge in $\bar{N}$, we can assume $|n| \leq 1$ for $n \in L^{-1} L$.

As usual, the conclusion follows if we show that the operator $\tilde{M}$ defined for $n_{1} \in L$ by

$$
\tilde{M} f\left(n_{1}\right)=\sup _{\substack{H \in V \\ H \text { large }}} \mathscr{P}_{0}|f|\left(n_{1} \exp H\right)
$$

is of weak type $(1,1)$ in $L$. We now use (2.4). Lemma 3.3 implies that $\psi(H) \sim|H|^{\sigma}$ for $H \in V$, where $|H|^{2}=\langle H, H\rangle$ and $\sigma=\operatorname{card} \Sigma_{0}^{+}$. As a result, the operator

satisfies $\tilde{M} f \leq C M f$ in $L$.

$$
M f=\sup _{\substack{H \in V \\ H \text { large }}}|H|^{-\sigma}|f| * \tilde{P}_{H}^{1 / 2}
$$

The following lemma can be seen as a stronger form of (5.1).

LEMMA 5.3. If $H \in \mathfrak{a}_{+}$with $H \geq 2^{k} \rho, k \in \mathbf{N}$, then for $\nu=0,1, \ldots, k$

$$
e^{2\langle\rho, H\rangle} \int_{e_{k-\nu}<|n|<1} P\left(n^{-H}\right)^{1 / 2} d n \leq C 2^{-\nu} \psi(H) .
$$


Proof. For $m=1, \ldots, 2^{\nu}$, we transform $n \rightarrow n^{-m 2^{k-\nu} \rho}$ and obtain

$$
\begin{array}{rl}
e^{2\langle\rho, H\rangle} \int_{e_{k-\nu}<|n|<1} & P\left(n^{-H}\right)^{1 / 2} d n \\
\leq & e^{2\left\langle\rho, H+m 2^{k-\nu} \rho\right\rangle} \int_{e_{k-\nu}^{m+1}<|n|<e_{k-\nu}^{m}} P\left(n^{-H-m 2^{k-\nu} \rho}\right)^{1 / 2} d n \\
\leq & e^{4\langle\rho, H\rangle} \int_{e_{k-\nu}^{m+1}<|n|<e_{k-\nu}^{m}} P\left(n^{-2 H}\right)^{I / 2} d n,
\end{array}
$$

because of Lemma 3.2. Now we can sum in $m$ and argue as when proving (5.1). The lemma follows.

We estimate the operator $M$. Any large $H \in V$ is smaller than some $2^{k} \rho, k \in \mathbf{N}$, with $|H| \sim\left|2^{k} \rho\right|$. By Lemma 3.2, it is therefore enough to take $H=2^{k} \rho, k=1,2, \ldots$, in the definition of $M$. For $0 \leq \nu \leq k$, we set

$$
K_{h}^{\nu}(n)=2^{-k \sigma} e^{2^{k+1}\langle\rho, \rho\rangle} \tilde{P}\left(n^{-2^{k} \rho}\right)^{1 / 2} \chi_{e_{k-\nu}<|n|<e_{k-\nu-1}},
$$

except that $e_{k}$ is replaced by 0 . Let

$$
M^{\nu} f=\sup _{k \geq \nu}|f| * K_{k}^{\nu}, \quad \nu=0,1, \ldots
$$

so that

$$
M f \leq C \sum_{\nu=0}^{\infty} M^{\nu} f .
$$

We shall apply Lemma 3.1 to each $M^{\nu}$. Fix $\nu$ and set $T_{k} f=$ $|f| * K_{k+\nu+1}^{\nu}$ for $k=-1,0, \ldots$ Lemma 5.3 gives assumption (i) with $C_{1}=C 2^{-\nu}$. Further, (ii) is clear. To verify (iii') with $N=\nu+1$, we must estimate $\left(K_{k+\nu+1}^{\nu}\right)^{*}$ and thus $\tilde{P}\left(\left(n n^{\prime}\right)^{-2^{k+\nu+1} \rho}\right)^{1 / 2}$ with $\left|n^{\prime}\right| \leq e_{k+\nu+1}$. But then $\left|\left(n^{\prime}\right)^{-2^{k+\nu+1} \rho}\right| \leq 1$, and we can apply Harnack's inequality (2.1) to $\tilde{P}$, since $\tilde{P}(n)=P_{\rho} \delta(n)$, where $\delta$ is a unit point mass at $e M \in K / M$. This gives

$$
\tilde{P}\left(\left(n n^{\prime}\right)^{-2^{k+\nu+1} \rho}\right) \leq C \tilde{P}\left(n^{-2^{k+\nu+1} \rho}\right) .
$$

Since also $\operatorname{supp}\left(K_{k+\nu+1}^{\nu}\right)^{*} \subset B\left(C e_{k}\right) \backslash B\left(e_{k+1} / C\right)$, Lemma 5.3 implies

$$
\int\left(K_{k+\nu+1}^{\nu}\right)^{*} d n \leq C \int K_{k+\nu+1}^{\nu} d n \leq C 2^{-\nu} .
$$

Lemma 3.1 now shows that $M^{\nu}$ is of weak type $(1,1)$, with constant at most $C(\nu+1) 2^{-\nu}$. This means that the series in (5.3) can be summed in weak $L^{1}$, so that $M$ and $\tilde{M}$ are of weak type $(1,1)$. Theorem 5.1 is proved. 
Next, let $X$ be of rank 1 . We let $\alpha$ and $2 \alpha$ be the positive roots and $m_{\alpha}>0$ and $m_{2 \alpha} \geq 0$ their multiplicities. The root space decomposition of $\overline{\mathfrak{n}}$ is $\overline{\mathfrak{n}}=\mathfrak{g}_{-\alpha} \oplus \mathfrak{g}_{-2 \alpha}$. In $X$ we choose the invariant metric associated with the Killing form in $\mathfrak{p}$ and denote by $d$ the corresponding distance. As usual, $\mathfrak{p}$ is the eigenspace of the Cartan involution in the Lie algebra of $G$ with eigenvalue -1 . Fix $H_{0} \in a_{+}$and choose an associated gauge in $\bar{N}$. Let $r_{k M}$ for $k \in K$ be the ray $\left\{k \exp \left(t H_{0}\right) \cdot o: t \geq 0\right\}$.

THEOREM 5.4. Let $\operatorname{rank} X=1$. Take $f \in L^{1}(K / M)$, and set $q=$ $1 /\left\langle 2 \rho, H_{0}\right\rangle$ and $\kappa=\sqrt{2 m_{\alpha}+8 m_{2 \alpha}} /\left(m_{\alpha}+2 m_{2 \alpha}\right)$. Then for almost all $n_{1} \in \bar{N}$

$$
\mathscr{P}_{0} f\left(n_{1} \exp \left(t H_{0}\right) n \cdot x\right) \rightarrow f\left(k\left(n_{1}\right) M\right)
$$

as $t \rightarrow+\infty$ and $n$ stays in the ball $B\left(C t^{q}\right)$ and $x$ in a compact subset of $X$, for any fixed $C$. Equivalently, $\mathscr{P}_{0} f(x) \rightarrow f(k M)$ as $x$ approaches $K / M$ while satisfying $d\left(x, r_{k M}\right) \leq \kappa \log d(x, o)+C$, for almost all $k M \in K / M$. This holds for no larger values of $q$ and $\kappa$.

As shown below, the two descriptions of the approach to the boundary in this theorem are equivalent in the following sense. Fix $n_{1} \in \bar{N}$. Given a constant $C_{1}<\infty$ and a compact set $D \subset X$, there exists $C_{2}<\infty$ such that

$$
d\left(x, r_{k\left(n_{1}\right) M}\right) \leq \kappa \log d(x, o)+C_{2}
$$

for all

(5.6) $x=n_{1} \exp \left(t H_{0}\right) n \cdot x^{\prime}$ with $t$ large, $|n| \leq C_{1} t^{q}$, and $x^{\prime} \in D$.

Conversely, any point $x$ far from $o$ satisfying (5.5) can be written as (5.6), where $C_{1}$ is determined by $C_{2}$ and $D=\{o\}$.

When $X$ is the disk $\mathbf{D}$, Theorem 5.4 is equivalent to the result from [16]. For this space $\kappa=\sqrt{2}$, which will agree with (4.6) if the hyperbolic metric is properly normalized.

Proof of Theorem 5.4. We first prove (5.4) in a rather sketchy way, since this is easy. One has

$$
P(n) \sim(1+|n|)^{-4\left\langle\rho, H_{0}\right\rangle},
$$

see Helgason [6], Theorem IX.3.8 p. 414, and $\psi(H) \sim|H|$ for large $H$. As in the preceding proof, we see that we need only take $f \in L^{1}(\bar{N})$ of compact support. Then we must estimate the convolution of $|f|$ with the 
kernel

$$
n \rightarrow t^{-1} e^{2\left\langle\rho, t H_{0}\right\rangle} \sup _{\left|n^{\prime}\right| \leq C t^{q}} \tilde{P}\left(n^{-t H_{0}} n^{\prime}\right)^{1 / 2} .
$$

If $\left|n^{-t H_{0}}\right|>C_{0} t^{q}$ and $C_{0}$ is large, one has $\left|n^{-t H_{0}}\right| \sim\left|n^{-t H_{0}} n^{\prime}\right|$. Because of (5.7), the factor $n^{\prime}$ can then be deleted from (5.8). The preceding proof, or Michelson [12], Theorem 2.2 (ii), now allows us to control the convolution with this part of the kernel.

For $\left|n^{-t H_{0}}\right| \leq C_{0} t^{q}$, we estimate $\tilde{P}$ by 1 in (5.8). These $n$ belong to the ball $B\left(C_{0} t^{q} e^{-t}\right)$. We simply obtain means of $|f|$ in translates of this ball, and thus a standard maximal function. This gives (5.4). To see that the given value of $q$ is sharp, one uses approximated point masses.

In order to prove the equivalence, we let $n_{1} \in \bar{N}$. Write

$$
n_{1} \exp \left(t H_{0}\right)=k\left(n_{1}\right) \exp \left(t H_{0}\right) \exp \left(H\left(n_{1}\right)\right) n\left(n_{1}\right)^{-t H_{0}},
$$

where the last two factors stay in a compact set as $t$ takes large values. Thus the curve $r_{n_{1}}: t \rightarrow n_{1} \exp \left(t H_{0}\right) \cdot o, t \geq 0$, stays at a bounded distance from $r_{k\left(n_{1}\right) M}$ and conversely, so that $\left|d\left(x, r_{n_{1}}\right)-d\left(x, r_{k\left(n_{1}\right) M}\right)\right| \leq$ $C$ for any $x$. This means that we can use $r_{n_{1}}$ instead of $r_{k\left(n_{1}\right) M}$ in (5.5). Translating everything by $n_{1}^{-1}$, we may assume $n_{1}=e$ since $d(x, o)-$ $d\left(n_{1}^{-1} x, o\right)$ stays bounded. Further, we can take $D=\{o\}$, because the point $x$ in (5.6) will move a bounded distance if $x^{\prime}$ is replaced by $o$. We can also normalize $H_{0}$ so that $\alpha\left(H_{0}\right)=1$. Notice that $\kappa=q\left|H_{0}\right|$.

The geodesic $A \cdot o$ contains $r_{e}$. We first estimate the distance to $A \cdot o$ from $x=\exp \left(t H_{0}\right) n \cdot o$ or, equivalently, from $n \cdot o$. Let $\beta=\log _{+}|n|$.

Lemma 5.5. $|d(n \cdot o, A \cdot o)-| H_{0}|\beta| \leq C$.

Proof. Consider first the path $s \rightarrow n \exp \left(-s H_{0}\right) \cdot o, 0 \leq s \leq \beta$, starting at $n \cdot o$ and of length $\left|H_{0}\right| \beta$. The distance from its endpoint $\exp \left(-\beta H_{0}\right) n^{\beta H_{0}} \cdot o$ to the point $\exp \left(-\beta H_{0}\right) \cdot o \in A \cdot o$ is at most $C$, because $\left|n^{\beta H_{0}}\right|=1$. It follows that $d(n \cdot o, A \cdot o) \leq\left|H_{0}\right| \beta+C$.

It remains to prove that any curve $\gamma$ from $n \cdot o$ to $A \cdot o$ has length at least $\left|H_{0}\right| \beta-C$. We can write $\gamma$ as

$$
\gamma(s)=n(s) \exp \left(-t(s) H_{0}\right) \cdot o, \quad 0 \leq s \leq L,
$$

where $n(s)$ and $t(s)$ take values in $\bar{N}$ and $\mathbf{R}$, respectively. Clearly $n(0)=n, t(0)=0$, and $n(L)=e$. Since we need only consider geodesics, we may assume that $\gamma$ is $C^{\infty}$ and that $n^{\prime}(s) \in \overline{\mathfrak{n}}$ never vanishes. This is because a geodesic parallel to the geodesic $n_{1} A \cdot o$ at some point would coincide with $n_{1} A \cdot o$. 
The lifted curve $n(s) \exp \left(-t(s) H_{0}\right)$ in $G$ has a tangent vector at $s$ given by the left-invariant field, i.e., the $g$ vector,

$$
-t^{\prime}(s) H_{0}+\operatorname{ad}\left(t(s) H_{0}\right) n^{\prime}(s) \text {. }
$$

If $n^{\prime}(s)=Y(s)+Z(s) \in \mathfrak{g}_{-\alpha} \oplus \mathfrak{g}_{-2 \alpha}$, this vector equals

$$
-t^{\prime}(s) H_{0}+e^{-t(s)} Y(s)+e^{-2 t(s)} Z(s) \text {. }
$$

The properties of the Killing form then imply that

$$
\left|\gamma^{\prime}(s)\right|^{2}=t^{\prime}(s)^{2}\left|H_{0}\right|^{2}+e^{-2 t(s)}|Y(s)|^{2}+e^{-4 t(s)}|Z(s)|^{2} \text {. }
$$

Here the bars $|\cdot|$ denote Euclidean norms in $\mathfrak{a}, \mathfrak{g}_{-\alpha}$, and $\mathfrak{g}_{-2 \alpha}$, respectively.

If $t(s)>\beta$ for some $s$, the desired lower estimate for the length of $\gamma$ is clear from (5.9). Assume thus $t \leq \beta$ in $[0, L]$. Then

$$
\left|\gamma^{\prime}(s)\right|^{2} \geq t^{\prime}(s)^{2}\left|H_{0}\right|^{2}+e^{-2 t(s)}\left(|Y(s)|^{2}+e^{-2 \beta}|Z(s)|^{2}\right) .
$$

Now reparametrize the curve so that $|Y(s)|^{2}+e^{-2 \beta}|Z(s)|^{2} \equiv\left|H_{0}\right|^{2}$ and thus

$$
\left|\gamma^{\prime}(s)\right|^{2}=\left|H_{0}\right|^{2}\left(t^{\prime}(s)^{2}+e^{-2 t(s)}\right)
$$

This parenthesis leads us to the curve $\tau(s)=s+i e^{-t(s)}, 0 \leq$ $s \leq L$, in the upper half-plane $\{y>0\}$ with the hyperbolic metric $y^{-2}\left(d x^{2}+d y^{2}\right)$. Indeed, $\left|\tau^{\prime}(s)\right|^{2}=t^{\prime}(s)^{2}+e^{-2 t(s)}$. Now $\tau$ connects $\tau(0)=i$ to the line $l=\{x=L\}$. The shortest curve connecting $i$ and $l$ is an arc of a Euclidean circle hitting $l$ and the real axis at right angles. Its hyperbolic length is greater than $\log L$. The length of $\gamma$ is thus greater than $\left|H_{0}\right| \log L$.

To estimate $L$, consider the curve $s \rightarrow n(s)^{\beta H_{0}} \cdot o, 0 \leq s \leq L$, in $X$. It goes from the point $n^{\beta H_{0}} \cdot o$ of the compact set $\{\tilde{n} \cdot o:|\tilde{n}|=1\}$ to $o$. Therefore, its length is bounded below. But this length is by the above

$$
\int_{0}^{L} e^{-\beta}\left|H_{0}\right| d s=\left|H_{0}\right| e^{-\beta} L \text {. }
$$

It follows that $L \geq c e^{\beta} /\left|H_{0}\right|, c>0$, so that the length of $\gamma$ is at least $\left|H_{0}\right| \beta-C$. Lemma 5.5 is proved.

Roughly speaking, this lemma means the following. To reach $A \cdot O$ from $n \cdot o$, move first "upwards" (in the negative $A$ direction) until the "horizontal" distance (in $\bar{N}$ ) to $A \cdot o$ is at most 1 . Then move horizontally. Further, $\exp \left(-\beta H_{0}\right) \cdot o$ is a closest point in $A \cdot o$, up to an additive constant in the distance. A consequence is that

$$
\left|d\left(\exp \left(t H_{0}\right) n \cdot o, o\right)-\right| H_{0}|| t-\beta|| \leq\left|H_{0}\right| \beta+C,
$$

as one sees by going via $\exp \left((t-\beta) H_{0}\right) \cdot o$. 
To prove that (5.6) implies (5.5), let $x=\exp \left(t H_{0}\right) n \cdot o$ with $|n| \leq C t^{q}$ and $t>0$ large. Then $\beta \leq q \log t+C$, and (5.10) implies that $\log d(x, o)$ $>\log t-C$. These inequalities and Lemma 5.5 yield (5.5).

Conversely, assume $d\left(x, r_{e}\right) \leq \kappa \log d(x, o)+C$ with $d(x, o)$ large, and write $x=\exp \left(t H_{0}\right) n \cdot o$. Because of (5.10),

$$
d(x, o) \leq C(|t|+\beta)+C .
$$

Combining this with the lower estimate of Lemma 5.5, we get

$$
\left|H_{0}\right| \beta \leq \kappa \log _{+}(|t|+\beta)+C \text {. }
$$

If $|t|$ is not large, this implies $\beta \leq C$ and $d(x, o) \leq C$ which is excluded. If $|t|>C$, observe that $\beta<|t|$. When $t>C$, we obtain $\beta \leq q \log t+C$ so that $|n| \leq C t^{q}$, and (5.6) follows. Finally if $t<-C$, then $d\left(x, r_{e}\right)$ is at least

$$
\begin{aligned}
d\left(\exp \left((t-\beta) H_{0}\right) \cdot o, r_{e}\right)-d\left(x, \exp \left((t-\beta) H_{0}\right) \cdot o\right) & \\
& \geq\left|H_{0}\right|(|t|+\beta)-\left|H_{0}\right| \beta-C=\left|H_{0}\right| t-C .
\end{aligned}
$$

This last quantity is not much smaller than $d(x, o)$, a contradiction which ends the proof of the equivalence.

Since the optimality of $q$ implies that of $\kappa$, the proof of Theorem 5.4 is complete.

6. Admissible convergence in $\mathrm{SL}(3, \mathbf{R}) / \mathrm{SO}(3)$. In this section and the next, $X$ will be $G / K=\operatorname{SL}(3, \mathbf{R}) / \mathrm{SO}(3)$. As a we take the set of diagonal matrices in $\mathfrak{g}=\{$ traceless $3 \times 3$ matrices $\}$. Denoting

$$
H=\left[\begin{array}{ccc}
h_{1} & 0 & 0 \\
0 & h_{2} & 0 \\
0 & 0 & h_{3}
\end{array}\right] \in \mathfrak{a}
$$

we define $a_{+}$by the condition $h_{1}>h_{2}>h_{3}$. Any $H \in \mathfrak{a}$ is then determined by the values of the two simple positive roots, $\alpha(H)=h_{1}-h_{2}$ and $\beta(H)=h_{2}-h_{3}$. The positive roots are $\alpha, \beta$, and $\alpha+\beta$. Thus $H$ is also determined by $\delta=e^{-\alpha(H)}$ and $\varepsilon=e^{-\beta(H)}$, and $H \rightarrow+\infty$ corresponds to $\delta, \varepsilon \rightarrow 0$. The group $\bar{N}$ consists of the matrices

$$
n=\left[\begin{array}{lll}
1 & 0 & 0 \\
x & 1 & 0 \\
z & y & 1
\end{array}\right], \quad x, y, z \in \mathbf{R}
$$

and we write simply $n=(x, y, z)$. Then $n^{H}=(\delta x, \varepsilon y, \delta \varepsilon z)$. The Poisson kernel in $\bar{N}$ is given by

$$
P(n)=\frac{1}{\left(1+x^{2}+z^{2}\right)\left(1+y^{2}+(z-x y)^{2}\right)} .
$$


This can be seen via a straightforward calculation and generalizes to $\mathrm{SL}(n, \mathbf{R}) / \mathrm{SO}(n)$, see Bhanu Murthy [3]. By Lemma 3.3, or Herz [8],

$$
\psi(H) \sim \log 1 / \delta \log 1 / \varepsilon \log 1 / \delta \varepsilon
$$

for small $\delta, \varepsilon$. Observe finally that the map

$$
(x, y, z) \rightarrow(-y,-x, x y-z)
$$

is an automorphism of $\bar{N}$ and preserves $P(n)$.

THEOREM 6.1. Let $p>1$. If $X=\mathrm{SL}(3, \mathbf{R}) / \mathrm{SO}(3)$ and $f \in L^{p}(K / M)$, then $\mathscr{P}_{0} f$ converges admissibly to f a.e. in $K / M$.

The method of proof below is based on products of one-dimensional maximal operators. In a simpler form, it also applies to $\mathscr{P}_{\lambda} f, \lambda \in \mathfrak{a}_{+}$, and gives a shorter proof of the main result in [17] for this $X$. However, it does not generalize to an arbitrary symmetric space.

Proof. Theorem 5.1 gives the convergence of $\mathscr{P}_{0} f$ for continuous $f$. As in the proof of that theorem, we can therefore take $f \in L^{1}(\bar{N})$ with support in a compact set $L$, and estimate the corresponding maximal function

$$
M f=\sup _{H \text { large }} \psi(H)^{-1}|f| * \tilde{P}_{H}^{1 / 2}
$$

in $L$. Assume $f \geq 0$. Clearly, $f * \tilde{P}_{H}^{1 / 2}\left(n_{1}\right)=\int f\left(n_{1} n^{H}\right) P(n)^{1 / 2} d n$, and for $n_{1} \in L$ we need only integrate over those $n$ satisfying $n^{H} \in L^{-1} L$. Hence with $n_{1}=\left(x_{1}, y_{1}, z_{1}\right)$, (6.3) $\quad \operatorname{Mf}\left(x_{1}, y_{1}, z_{1}\right)$

$$
\begin{aligned}
\leq & \sup _{\delta, \varepsilon \text { small }} \frac{C}{\log 1 / \delta \log 1 / \varepsilon \log 1 / \delta \varepsilon} \\
& \times \int \frac{f\left(x_{1}+\delta x, y_{1}+\varepsilon y, z_{1}+\delta \varepsilon z+\delta x y_{1}\right)}{\left(1+x^{2}+z^{2}\right)^{1 / 2}\left(1+y^{2}+(z-x y)^{2}\right)^{1 / 2}} d x d y d z,
\end{aligned}
$$

the integral taken over $\{|x| \leq C / \delta,|y| \leq C / \varepsilon,|z| \leq C / \delta \varepsilon\}$. Write this integral as

$$
\int_{|z|<|x y| / 2}+\int_{|z-x y|<|x y| / 2}+\int_{\text {remaining } x, y, z}=\mathrm{I}+\mathrm{II}+\mathrm{III} .
$$

In I, we can estimate $P(n)^{1 / 2}$ by $C(1+|x|+|z|)^{-1}(1+|y|+|x y|)^{-1}$. This expression is even in $z$ and decreasing in $|z|$, and its integral with respect to $z$ in $|z|<|x y| / 2$ is at most

$$
C\left(1+\log _{+}|y|\right)(1+|y|+|x y|)^{-1} \leq C \log 1 / \varepsilon(1+(1+|x|)|y|)^{-1} .
$$


Thus we can integrate with respect to $z$ in I and estimate the result by means of the standard one-dimensional maximal operator taken in the $z$ direction. This operator is denoted by $M_{0,0,1}$. This means that

$$
\mathrm{I} \leq C \log 1 / \varepsilon \int_{\substack{|x| \leq C / \delta \\|y| \leq C / \varepsilon}} \frac{M_{0,0,1} f\left(x_{1}+\delta x, y_{1}+\varepsilon y, z_{1}+\delta x y_{1}\right)}{1+(1+|x|)|y|} d x d y .
$$

The $y$ integral here can similarly be estimated by means of the maximal operator $M_{0,1,0}$, so that

$$
\begin{aligned}
\mathrm{I} \leq & C \log 1 / \varepsilon \int_{|x|<C / \delta} \frac{\log 1 / \varepsilon+\log (1+|x|)}{1+|x|} \\
& \times M_{0,1,0} M_{0,0,1} f\left(x_{1}+\delta x, y_{1}, z_{1}+\delta x y_{1}\right) d x .
\end{aligned}
$$

For the remaining integration, we use the maximal operator in the direction $\left(1,0, y_{1}\right)$, getting

$$
\begin{aligned}
I \leq & C \log 1 / \varepsilon\left(\log 1 / \varepsilon \log 1 / \delta+(\log 1 / \delta)^{2}\right) \\
& \times M_{1,0, y_{1}} M_{0,1,0} M_{0,0,1} f\left(x_{1}, y_{1}, z_{1}\right) .
\end{aligned}
$$

The logarithmic factors here are controlled by those in (6.3).

We now claim that the operator $M_{1,0, y_{1}}$ is bounded on $L^{p}\left(\mathbf{R}^{3}\right)$. Indeed, for each fixed value of $y_{1}$, we have a maximal operator along a fixed direction in the $x z$ plane. Thus,

$$
\iint\left(M_{1,0, y_{1}} g\left(x_{1}, y_{1}, z_{1}\right)\right)^{p} d x_{1} d z_{1} \leq C \iint\left|g\left(x, y_{1}, z\right)\right|^{p} d x d z
$$

for $g \in L^{p}\left(\mathbf{R}^{3}\right)$, uniformly in $y_{1}$. Integration in $y_{1}$ now gives the claimed boundedness. As a result, that part of $M$ which corresponds to $\mathrm{I}$ is bounded on $L^{p}$.

The term II can be treated like I after the transformation (6.2), which interchanges $\delta$ and $\varepsilon$.

In III, we have $|z-x y|>|x y| / 2$ and $|z|>|x y| / 2$. Therefore, the argument used for I, and that of II, will apply. But in both arguments, the $z$ integration will now produce a factor $\log 1 / \delta \varepsilon$ instead of $\log 1 / \varepsilon$ or $\log 1 / \delta$. Since $\log 1 / \delta \varepsilon \sim \max (\log 1 / \delta, \log 1 / \varepsilon)$, one argument or the other will give the right estimate for III. We conclude that $M$ is bounded on $L^{p}$, and Theorem 6.1 is proved.

More algebraically, one could also have used the fact that the onedimensional maximal operators in this proof are associated with oneparameter subgroups. 
7. Improved restricted convergence in $\operatorname{SL}(3, \mathbf{R}) / \mathrm{SO}(3)$. We use the notation from $\S 6$. Fix $H_{0} \in \mathfrak{a}_{+}$and write $\alpha$ and $\beta$ for $\alpha\left(H_{0}\right)$ and $\beta\left(H_{0}\right)$, which are positive numbers.

TheOREM 7.1. Let $X=\operatorname{SL}(3, \mathbf{R}) / \operatorname{SO}(3)$ and take $f \in L^{1}(K / M)$. Set $q=2 /(\alpha+\beta+\max (\alpha, \beta))$. Then for a.a. $n_{1} \in \bar{N}$

$$
\mathscr{P}_{0} f\left(n_{1} \exp \left(t H_{0}\right) n \cdot x\right) \rightarrow f\left(k\left(n_{1}\right) M\right)
$$

as $t \rightarrow+\infty,|n| \leq C t^{q}$, and $x \in X$ stays in a compact set. This holds for no larger value of $q$.

Formally, this result is like the first part of Theorem 5.4. Notice, however, that the widening of the convergence region caused by the factor $n$ is now only in certain directions. Indeed, as $t$ and $n$ vary, the points $n_{1} \exp \left(t H_{0}\right) n \cdot o$ describe a set whose dimension is smaller than that of $X$.

For the proof of Theorem 7.1, we need a proposition of independent interest. Consider for $\mu>0$ the kernel

$$
P_{\mu, 0}^{t}(n)=\frac{t^{-1} e^{2 \alpha t+2 \beta t}}{\left(1+e^{\alpha t}|x|+e^{\alpha t+\beta t}|z|\right)^{1+\mu}\left(1+e^{\beta t}|y|+e^{\alpha t+\beta t}|z-x y|\right)}
$$

where $t>1$ and $n=(x, y, z) \in \bar{N}$. Similarly, $P_{0, \mu}^{t}$ will denote the kernel obtained by interchanging the exponents $1+\mu$ and 1 of the two factors in the denominator.

The automorphism (6.2) interchanges $P_{\mu, 0}^{t}$ and $P_{0, \mu}^{t}$. These kernels, or rather $\tilde{P}_{\mu, 0}^{t}$ and $\tilde{P}_{0, \mu}^{t}$, arise from normalized $\lambda$-Poisson integrals $\mathscr{P}_{\lambda}\left(n_{1} \exp \left(t H_{0}\right)\right)$ with a nonzero $\lambda$ on the boundary of $\mathfrak{a}_{+}$. This is why the $P_{\mu, 0}^{t}, t>0$, form a family of normalized dilations of one function if the factor $t^{-1}$ is deleted. Also,

$$
P_{\mu, 0}^{t}\left(n^{\prime} n\right) \sim P_{\mu, 0}^{t}(n)
$$

for $n^{\prime} \in B\left(C e^{-t}\right)$ and any $n$. This is seen from Harnack's inequality or directly from the definition of the kernel. Let

$$
M_{\mu, 0} f=\sup _{t \text { large }}|f| * \tilde{P}_{\mu, 0}^{t}, \quad f \in L^{1}(\bar{N}),
$$

and similarly for $M_{0, \mu}$. These are the maximal functions corresponding to restricted convergence of $\mathscr{P}_{\lambda} f$ for $\lambda \in \partial a_{+} \backslash\{0\}$. A recent result by van den Ban and Schlichtkrull [2, Cor. 16.6] implies that this convergence holds for continuous $f$. 
Proposition 7.2. If $L \subset \bar{N}$ is compact, $M_{\mu, 0}$ and $M_{0, \mu}$ are of weak type $(1,1)$ in $L$.

This has the following consequence.

TheOrem 7.3. Let $X=\operatorname{SL}(3, \mathbf{R}) / \operatorname{SO}(3)$ and take $\lambda \in \partial a_{+} \backslash\{0\}$ and $f \in L^{1}(K / M)$. Then $\mathscr{P}_{\lambda} f$ converges restrictedly to $f$ a.e. in $K / M$.

Proof of Proposition 7.2. Because of the map (6.2), we need only work with $M_{\mu, 0}$. The idea of the proof is to apply Lemma 3.1, after some elementary reductions. Write temporarily $\tilde{x}, \tilde{y}$, and $\tilde{z}$ for $e^{\alpha t} x, e^{\beta t} y$, and $e^{\alpha t+\beta t} z$, respectively.

First of all, it is enough to treat that part of $P_{\mu, 0}^{t}$ defined by

$$
|\tilde{x}|+|\tilde{z}| \leq e^{\eta t}
$$

for any fixed $\eta>0$. Indeed, if this inequality is false, $(1+|\tilde{x}|+|\tilde{z}|)^{\mu}>$ $t^{2} / C$. Then $P_{\mu, 0}^{t}$ is dominated by the kernel defining $\mathscr{P}_{0} f$, which is controlled by Theorem 5.1.

Similarly, we can restrict $P_{\mu, 0}^{t}$ by

$$
10(1+|\tilde{x}|+|\tilde{z}|)<1+|\tilde{y}|+|\tilde{z}-\tilde{x} \tilde{y}|,
$$

This is because that part of $P_{\mu, 0}^{t}$ corresponding to the opposite inequality can be estimated by

$$
\frac{C e^{2 \alpha t+2 \beta t}}{(1+|\tilde{x}|+|\tilde{z}|)^{1+\mu / 2}(1+|\tilde{y}|+|\tilde{z}-\tilde{x} \tilde{y}|)^{1+\mu / 2}} .
$$

This is essentially the kernel obtained for $\mathscr{P}_{\lambda} f$ with some $\lambda \in \mathfrak{a}_{+}$. But such $\mathscr{P}_{\lambda} f$ are controlled by the results of Stein [18] or Sjögren [17], §7.

We next verify that it is enough to prove the proposition with $P_{\mu, 0}^{t}$ replaced by the kernel

$$
\frac{t^{-1} e^{2 \alpha t+2 \beta t}}{(1+|\tilde{x}|)^{1+\gamma}(1+|\tilde{z}|)^{1+\gamma}(1+|\tilde{y}|)},
$$

for $\gamma>0$.

In case $|\tilde{y}| \leq|\tilde{z}-\tilde{x} \tilde{y}|$, we see from (7.3) that $|\tilde{z}|<|\tilde{z}-\tilde{x} \tilde{y}| / 2$, which implies $|\tilde{z}-\tilde{x} \tilde{y}| \sim|\tilde{x} \tilde{y}|$. Hence, $|\tilde{x}|<|\tilde{x} \tilde{y}|$ because of (7.3), so that $|\tilde{y}|>1$ and $1+|\tilde{y}|+|\tilde{z}-\tilde{x} \tilde{y}| \sim(1+|\tilde{x}|)|\tilde{y}|$. Then $P_{\mu, 0}^{t}$ is dominated by (7.4), with $\gamma=\mu / 2$.

In the opposite case $|\tilde{y}|>|\tilde{z}-\tilde{x} \tilde{y}|$, (7.3) implies $|\tilde{z}|<|\tilde{y}|$. Then $|\tilde{x} \tilde{y}|$ $<2|\tilde{y}|$, so that $|\tilde{x}|<2$ and (7.4) again dominates $P_{\mu, 0}^{t}$. 
We are thus led to integrals of $f\left(n_{1} n\right)$ against the kernel (7.4) restricted by (7.2). Given a large $t$, choose an integer $k$ so that $2^{k-1}<t \leq$ $2^{k}$. If $\eta$ is small, (7.2) then implies $|x| \leq e_{k-2}^{\alpha}$ and $|z| \leq e_{k-2}^{\alpha+\beta}$. Now we make a decomposition of (7.4) like that of $L_{t}$ in (4.3). We arrive at the kernel

$$
\sum_{\nu=0}^{k} \frac{2^{-k} e^{2 \alpha t+\beta t}}{\left(1+e^{\alpha t}|x|\right)^{1+\gamma}\left(1+e^{\alpha t+\beta t}|z|\right)^{1+\gamma}\left(e_{k-\nu}^{\beta}+|y|\right)} \chi_{k, \nu},
$$

where $\chi_{k, \nu}$ is the characteristic function of the set $\left\{|x| \leq e_{k-2}^{\alpha},|z| \leq e_{k-2}^{\alpha+\beta}\right.$, $\left.|y| \leq e_{k-\nu-1}^{\beta}\right\}$. Let $K_{t}^{\nu}(n)$ denote term number $\nu$ here, $n=(x, y, z)$, and set

$$
T_{k}^{\nu} f\left(n_{1}\right)=\sup _{2^{k-1}<t \leq 2^{k}} \int\left|f\left(n_{1} n\right)\right| K_{t}^{\nu}(n) d n, \quad k \geq \nu .
$$

By means of Lemma 3.1, we shall prove that the operator

$$
f \rightarrow \sup _{k \geq \nu} T_{k}^{\nu} f
$$

is for each $\nu$ of weak type $(1,1)$, with constant at most $C(\nu+1) 2^{-\nu}$. This will allow summation in $\nu$ and complete the proof.

Thus we must verify (i), (ii), (iii') and start with (i). Clearly,

$$
\begin{aligned}
T_{k}^{\nu} f\left(n_{1}\right) \leq & \sup _{t} 2^{-\nu} \int \frac{e^{2 \alpha t+\beta t} d x d z}{\left(1+e^{\alpha t}|x|\right)^{1+\gamma}\left(1+e^{\alpha t+\beta t}|z|\right)^{1+\gamma}} \\
& \times \int_{|y| \leq e_{k-\nu-1}^{\beta}} \frac{2^{\nu-k} f\left(n_{1} n\right)}{e_{k-\nu}^{\beta}+|y|} d y .
\end{aligned}
$$

The inner integral here is a convolution of $f$ and a measure which is independent of $t$ and of total mass at most $C$. This integral thus defines a bounded operator $L^{1} \rightarrow L^{1}$. The integration in $x$ and $z$ amounts to applying to $f$ a one-parameter two-dimensional maximal operator. As an operator in the plane, it is of weak type $(1,1)$, with a uniform constant. The fact that it acts here in the plane spanned by $\left(1,0, y_{1}\right)$ and $(0,0,1)$ in $\mathbf{R}^{3}$ causes no problem, cf. the operator $M_{1,0, y_{1}}$ in $\S 6$. Thus $T_{k}^{\nu}$ is of weak type $(1,1)$ with constant $O\left(2^{-\nu}\right)$, which is (i).

For (ii) we simply observe that $T_{k}^{\nu} f\left(n_{1}\right)$ is determined by the restriction of $f$ to $n_{1} B\left(e_{k-\nu-2}\right)$, if the gauge in $\bar{N}$ is suitably normalized.

Finally we must estimate

$$
\int\left(\tilde{K}_{t}^{\nu}\right)^{*} d n=\int \sup K_{t}^{\nu}\left(n^{\prime} n\right) d n,
$$


the supremum taken over $\left|n^{\prime}\right| \leq e_{k+C}$. If $n \in L$ and $C$ is large, the coordinates of $n^{\prime} n$ and $n$ here will differ by at most $e_{k}^{\alpha}, e_{k}^{\beta}$, and $e_{k}^{\alpha+\beta}$, respectively. The denominator in the expression for $K_{t}^{\nu}$ is then essentially the same at $n^{\prime} n$ and $n$. The support of $\sup K_{t}^{\nu}\left(n^{\prime} n\right)$ is only slightly larger than that of $K_{t}^{\nu}(n)$. Thus, $\tilde{K}_{t}^{\nu}$ and $\left(\tilde{K}_{t}^{\nu}\right)^{*}$ have the same $L^{1}$ bound $C 2^{-\nu}$, and (iii') is proved.

Now Lemma 3.1 applies with $C_{1}=2^{-\nu}$ and $N=\nu+C$. Proposition 7.2 is proved.

Proof of Theorem 7.1. As when proving Theorem 5.1, we take $0 \leq f \in$ $L^{1}(\bar{N})$ supported in a compact set $L$. Then we must estimate $\mathscr{P}_{0} f$ at the point $n_{1} \exp \left(t H_{0}\right) n$, or equivalently at

$$
g=n_{1} \exp \left((t+q \log t) H_{0}\right) n
$$

for $n_{1} \in L$, large $t$, and $|n| \leq C t^{q}$. One has $g=n_{1} n^{\prime} \exp \left((t+q \log t) H_{0}\right)$, where $\left|n^{\prime}\right| \leq C e^{-t}$. Then (2.4) implies

$$
\mathscr{P}_{0} f(g) \leq C \int f\left(n_{1} n^{\prime} n\right) t^{-3+2 \alpha q+2 \beta q} e^{2 \alpha t+2 \beta t} P\left(n^{-(t+q \log t) H_{0}}\right)^{1 / 2} d n,
$$

and here

$$
\begin{aligned}
& P\left(n^{-(t+q \log t) / I_{0}}\right)^{1 / 2} \\
& -\frac{1}{\left(1+t^{\alpha q} e^{\alpha t}|x|+t^{\alpha q+\beta q} e^{\alpha t+\beta t}|z|\right)\left(1+t^{\beta q} e^{\beta t}|y|+t^{\alpha q+\beta q} e^{\alpha t+\beta t}|z-x y|\right)}
\end{aligned}
$$

with $n=(x, y, z)$. By decomposing $\bar{N}$ into several subsets, we shall divide the integral in (7.5) into parts which are considered separately. The decomposition will depend on the relative sizes of the terms in the denominator of (7.6), with the powers of $t$ deleted. Parts 1 and 2 will mostly be handled by a method due to $\mathrm{T}$. Lyons (oral communication). The idea is to estimate $\mathscr{P}_{0} f(g)$, or parts of it, by $\mathscr{P}_{\lambda} f\left(g^{\prime}\right)$, where $\lambda$ may be different from 0 and $g^{\prime} \cdot o$ is a point further from the boundary than $g \cdot o$. We shall have $g^{\prime}=n_{1} n^{\prime} \exp \left(t H_{0}\right)$. For Part 3 , we use more direct estimates.

Part 1: $\max \left(1, e^{\alpha t}|x|\right) \leq e^{\alpha t+\beta t}|z|$ and $\max \left(1, e^{\beta t}|y|\right) \leq e^{\alpha t+\beta t}|z-x y|$. The kernel in (7.5) is dominated by

$$
\begin{aligned}
& t^{-3} \frac{1}{|z||z-x y|} \\
& \quad \leq \frac{C t^{-3} e^{2 \alpha t+2 \beta t}}{\left(1+e^{\alpha t}|x|+e^{\alpha t+\beta t}|z|\right)\left(1+e^{\beta t}|y|+e^{\alpha t+\beta t}|z-x y|\right)} .
\end{aligned}
$$


The corresponding part of $\mathscr{P}_{0} f(g)$ is thus dominated by

$$
\mathscr{P}_{0} f\left(n_{1} n^{\prime} \exp \left(t H_{0}\right)\right)=\mathscr{P}_{0} f\left(n_{1} \exp \left(t H_{0}\right) n^{\prime \prime}\right),
$$

where $n^{\prime \prime}$ stays in a compact set. This is controlled by Theorem 5.1.

Part 2: $\max \left(e^{\alpha t}|x|, e^{\alpha t+\beta t}|z|\right) \leq 1$. This will also include the case $\max \left(e^{\beta t}|y|, e^{\alpha t+\beta t}|z-x y|\right) \leq 1$, as seen via (6.2).

2a: $e^{\beta t}|y| \leq 1$. Now $(x, y, z) \in B\left(C e^{-t}\right)$, and this part of $\mathscr{P}_{0} f(g)$ is majorized by

$$
t^{-3+2 \alpha q+2 \beta q} e^{2 \alpha t+2 \beta t} \int_{n_{1} n^{\prime} B\left(C e^{-t}\right)} f(n) d n .
$$

Changing $C$, we have $n_{1} n^{\prime} B\left(C e^{-t}\right) \subset n_{1} B\left(C e^{-t}\right)$. Since $\left|B\left(C e^{-t}\right)\right| \sim$ $e^{-2 \alpha t-2 \beta t}$ and the exponent of $t$ in (7.7) is negative, the expression (7.7) is dominated by the mean value of $f$ in $n_{1} B\left(C e^{-t}\right)$. This leads to the standard maximal operator in $\bar{N}$, which is of weak type $(1,1)$.

2b: $e^{\beta t}|y|>1$. Here $e^{\alpha t+\beta t}|z-x y| \leq 2 e^{\beta t}|y|$, and we can estimate the kernel of (7.5) by

$$
\begin{aligned}
& \frac{C t^{-3+2 \alpha q+2 \beta q} e^{2 \alpha t+2 \beta t}}{\left(1+e^{\alpha t}|x|+e^{\alpha t+\beta t}|z|\right)^{2} t^{\beta q} e^{\beta t}|y|} \\
& \quad \leq \frac{C t^{-3+2 \alpha q+\beta q} e^{2 \alpha t+2 \beta t}}{\left(1+e^{\alpha t}|x|+e^{\alpha t+\beta t}|z|\right)^{2}\left(1+e^{\beta t}|y|+e^{\alpha t+\beta t}|z-x y|\right)} \\
& \quad=C t^{-2+2 \alpha q+\beta q} P_{1,0}^{t}(n) .
\end{aligned}
$$

Again, the exponent of $t$ is nonpositive. Proposition 7.2 gives the necessary estimate, since (7.1) takes care of the factor $n^{\prime}$ of (7.5).

Part 3: $\max \left(1, e^{\alpha t+\beta t}|z|\right) \leq e^{\alpha t}|x|$. This also takes care of the case $\max \left(1, e^{\alpha t+\beta t}|z-x y|\right) \leq e^{\beta t}|y|$.

3a: $e^{\beta t}|y| \leq 2$. We can assume that $e^{\alpha t+\beta t}|z-x y|>1$, since the contrary case is covered by Part 2. For such $n,(7.6)$ is at most $C t^{-2 \alpha q-\beta q} e^{-2 \alpha t-\beta t}|x|^{-1}|z-x y|^{-1}$. Let

$$
K_{t}(n)=\frac{C t^{-3+\beta q} e^{\beta t}}{\left(e^{-\alpha t}+|x|\right)\left(e^{-\alpha t-\beta t}+|z-x y|\right)} \chi_{|y| \leq 2 e^{-\beta t}} .
$$


That part of (7.5) corresponding to 3a can be estimated by

$$
\int f\left(n_{1} n\right) K_{t}\left(\left(n^{\prime}\right)^{-1} n\right) d n \leq \int f\left(n_{1} n\right) K_{t}^{*}(n) d n,
$$

where

$$
K_{t}^{*}(n)=\sup K_{t}\left(\left(n^{\prime}\right)^{-1} n\right)
$$

and the supremum is taken over $n^{\prime} \in B\left(C e^{-t}\right)$. Comparing the coordinates of $\left(n^{\prime}\right)^{-1} n$ and $n$, we see that

$$
K_{t}^{*}(n) \leq \frac{C t^{-3+\beta q} e^{\beta t}}{\left(e^{-\alpha t}+|x|\right)\left(e^{-\alpha t-\beta t}+|z-x y|\right)} \chi_{|y| \leq C e^{-\beta t} .}
$$

The automorphism (6.2) transforms $K_{t}^{*}(n)$ to

$$
\frac{t^{-3+\beta q} e^{\beta t}}{\left(e^{-\alpha t}+|y|\right)\left(e^{-\alpha t-\beta t}+|z|\right)} \chi_{|x|<C e^{-\beta t}}=L_{t}(n),
$$

say. We write

$$
\sup _{t} \int f\left(n_{1} n\right) L_{t}(n) d n \leq \sum_{k} \sup _{2^{k-1} \leq t \leq 2^{k}} \int f\left(n_{1} n\right) L_{t}(n) d n .
$$

For $2^{k-1} \leq t \leq 2^{k}$

$$
L_{t}(n) \leq C \frac{2^{-3 k+\beta q k} e^{\beta t}}{\left(e_{k}^{\alpha}+|y|\right)\left(e_{k}^{\alpha+\beta}+|z|\right)} \chi_{|x| \leq C e^{-\beta t}} .
$$

Here we have no $t$ dependence in the factors containing $y$ and $z$. Thus we can estimate $\int f\left(n_{1} n\right) L_{t}(n) d n$ for $2^{k-1} \leq t \leq 2^{k}$ by integrating first in these variables. This means convolving $f$ with a finite measure, which preserves $L^{1}$. The integral in $x$ can then be estimated by means of the standard maximal function, taken in the direction $\left(1,0, y_{1}\right)$. Thus, term number $k$ in the sum in (7.8) defines an operator of weak type (1,1). The associated constant is seen to be $O\left(2^{-3 k+\beta q k+k+k}\right)=O\left(2^{(\beta q-1) k}\right)$. Since $\beta q<1$, the sum in (7.8) will be in weak $L^{1}$, and we get an estimate which ends Part 3a.

3b: $e^{\beta t}|y|>2$. Now $|z-x y| \sim|x y|$, and the expression in (7.6) is bounded by $C t^{-2 \alpha q-\beta q} e^{-2 \alpha t-\beta t}|x|^{-1}|x y|^{-1}$. We proceed as in 3a and obtain kernels $K_{t}$ and $K_{t}^{*}$ with

$$
K_{t}^{*}(n) \leq C \frac{t^{-3+\beta q} e^{\beta t}}{\left(e^{-\alpha t}+|x|\right)^{2}\left(e^{\beta t}+|y|\right)} \chi_{t}(n),
$$


$\chi_{t}$ being the characteristic function of $\left\{e^{\alpha t+\beta t}|z| \leq C\left(1+e^{\alpha t}|x|\right)\right\}$. Assume again that $2^{k-1} \leq t \leq 2^{k}$. Then

$$
\begin{aligned}
K_{t}^{*}(n) & \leq C \sum_{j} 2^{-3 k+\beta q k} e^{\beta t}\left(2^{-j} e^{\alpha t}\right)^{2} \frac{1}{e_{k}^{\beta}+|y|} \chi_{t, j}(n) \\
& \equiv C \sum_{j} K_{t}^{(j)}(n),
\end{aligned}
$$

where $\chi_{t, j}$ is the characteristic function of $\left\{|x| \leq 2^{j} e^{-\alpha t},|z| \leq C 2^{j} e^{-\alpha t-\beta t}\right\}$ and $j$ ranges from 0 to an upper bound which is $O\left(2^{k}\right)$.

Let us estimate

$$
\sup _{2^{k-1} \leq t \leq 2^{k}} \int f\left(n_{1} n\right) K_{t}^{(j)}(n) d n .
$$

We integrate first in $y$ and obtain a convolution of $f$ with a finite measure. As before, the integral in $x$ and $z$ is then estimated by means of a one-parameter two-dimensional maximal operator. This gives a weak type $(1,1)$ estimate in $\mathbf{R}^{3}$ for the operator (7.10), and the associated constant is seen to be $O\left(2^{(\beta q-2) k}\right)$.

To sum in $j$, we use the addition theorem for the weak $L^{1}$ quasinorm $\|\cdot\|$ from Stein [18], §6. It says that

$$
\left\|\sum_{1}^{N} f_{i}\right\| \leq C \log N \sum_{1}^{N}\left\|f_{i}\right\|, \quad N \geq 2 .
$$

Thus the operator

$$
f \rightarrow \sup _{2^{k-1} \leq t \leq 2^{k}} \int f\left(n_{1} n\right) K_{t}^{*}(n) d n
$$

is of weak type $(1,1)$, with a constant which is $O\left(k 2^{(\beta q-1) k}\right)$. This allows us to sum in $k$ and finish Part $3 b$.

It only remains to verify that the value of $q$ is sharp. Let $m$ denote Lebesgue measure in $[-1,1]$ and set

$$
\mu=\delta \otimes m \otimes \delta .
$$

Here $\delta$ is the Dirac measure at $0 \in \mathbf{R}$, and the tensor product refers to the coordinates $(x, y, z)$ of $\bar{N}$. Take any $q>0$. At a point $n_{0}=\left(0, y_{0}, 0\right)$, $\left|y_{0}\right| \leq 1$, one has for large $t$

$$
\begin{aligned}
\mathscr{P}_{0} f\left(n_{0} \exp \left(t H_{0}\right)\right) & \sim t^{-3} e^{2 \alpha t+2 \beta t} \int_{|y| \leq 1} \frac{d y}{1+e^{\beta t}\left|y_{0}+y\right|} \\
& \sim t^{-2} e^{2 \alpha t+\beta t}
\end{aligned}
$$


Let $n_{1}=\left(x_{1}, y_{1}, z_{1}\right)$ be a point with $\left|x_{1}\right| \leq t^{\alpha q} e^{-\alpha t}, \quad\left|y_{1}\right| \leq 1$, and $\left|z_{1}-x_{1} y_{1}\right| \leq t^{\alpha q+\beta q} e^{-\alpha t-\beta t}$. Then the point $n^{\prime}=\left(-x_{1}, 0, x_{1} y_{1}-z_{1}\right)$ satisfies $n_{1} n^{\prime}=\left(0, y_{1}, 0\right)$, and $n=\left(n^{\prime}\right)^{-t H_{0}}$ stays in a ball $B\left(C t^{q}\right)$. Because of (7.11) we get

$$
\sup _{|n| \leq C t^{q}} \mathscr{P}_{0} \mu\left(n_{1} \exp \left(t H_{0}\right) n\right) \geq c t^{-2} e^{2 \alpha t+\beta t},
$$

for some $c>0$. The set of such $n_{1}$ has measure $8 t^{2 \alpha q+\beta q} e^{-2 \alpha t-\beta t}$. When $2 \alpha q+\beta q>2$, this is enough to disprove the weak type $(1,1)$ of the maximal function associated to $H_{0}$ and $q$. The symmetry in $\alpha$ and $\beta$ obtained from (6.2) now implies that the value of $q$ in the theorem is sharp for weak type $(1,1)$.

The measure $\mu$ leads to a counterexample to a.e. convergence by a rather standard method which we briefly describe. By approximating and contracting $\mu$, one constructs a sequence $\left(f_{k}\right)$ of nonnegative functions in $L^{1}(\bar{N})$ supported near $e$ such that $\sum \int f_{k} d n<\infty$ and for some $t_{k} \rightarrow+\infty$ and $\omega_{k} \rightarrow+\infty$

$$
\sup _{|n| \leq C t_{k}^{q}} \mathscr{P}_{0} f_{k}\left(n_{1} \exp \left(t_{k} H_{0}\right) n\right) \geq \omega_{k}
$$

on a set $A_{k}$. Moreover, $\Sigma\left|A_{k}\right|=+\infty$ and the $A_{k}$ are contained in a small neighborhood of 0 .

It is enough to take a small ball $B$ centered at $e$ and verify the following: Given $k^{\prime} \in \mathbf{N}$, there exist $k^{\prime \prime}>k^{\prime}$ and points $n_{k} \in B, k^{\prime}<k$ $\leq k^{\prime \prime}$, such that the union of translates $\bigcup\left\{n_{k} A_{k}: k^{\prime}<k \leq k^{\prime \prime}\right\}$ has measure at least $|B| / 2$. Indeed, then we can iterate this construction to form segments of a sequence $\left(n_{k}\right)_{1}^{\infty}$ such that the set of points belonging to infinitely many sets $n_{k} A_{k}$ has positive measure. The required counterexample $f$ will then be the sum of the corresponding translates of the $f_{k}$.

To make this construction, assume $n_{k}$ has been chosen for $k^{\prime}<k<j$ and that the set $M_{j-1}=\bigcup\left\{n_{k} A_{k}: k^{\prime}<k<j\right\}$ has measure smaller than $|B| / 2$. Considering the average of $\left|M_{j-1} \cap n A_{j}\right|$ as $n$ runs over $B$, we find an $n_{j}$ with $\left|M_{j-1} \cap n_{j} A_{j}\right| \leq\left|A_{j}\right| / 2$. Thus $\left|\left(n_{j} A_{j}\right) \backslash M_{j-1}\right| \geq\left|A_{j}\right| / 2$, and the rest is easy.

Theorem 7.1 is proved.

\section{REFERENCES}

[1] E. P. van den Ban, Asymptotic expansions and integral formulas for eigenfunctions on a semisimple Lie group. Thesis, Univ. of Utrecht, 1982.

[2] E. P. van den Ban and H. Schlichtkrull, Asymptotic expansions and boundary values of eigenfunctions on Riemannian symmetric spaces. To appear in J. Reine Angew. Math. 
[3] T. S. Bhanu Murthy, Plancherel's measure for the factor space $\operatorname{SL}(n ; R) / \operatorname{SO}(n ; R)$. Dokl. Acad. Nauk SSSR, 113 (1960), 503-506 (= Soviet Math. Dokl., 1 (1960), 860-862).

[4] M. de Guzmán, Real Variable Methods in Fourier Analysis, North-Holland, Amsterdam, 1981.

[5] Harish-Chandra, Spherical functions on a semisimple Lie group I. Amer. J. Math., 80 (1958), 241-310.

[6] S. Helgason, Differential Geometry, Lie Groups, and Symmetric Spaces, Academic Press, New York, 1978.

[7] Groups and Geometric Analysis. Integral Geometry, Invariant Differential Operators, and Spherical Functions, Academic Press, New York, 1984.

[8] C. Herz, The Poisson kernel for sl $(3, R)$, pp. 333-346 in Théorie du potentiel, ed. G. Mokobodzki and G. Pinchon, Lecture Notes in Mathematics 1096, Springer, 1984.

[9] A. W. Knapp and R. E. Williamson, Poisson integrals and semisimple groups, J. Analyse Math., 24 (1971), 53-76.

[10] A. Korányi, Harmonic Functions on Symmetric Spaces, pp. 379-412 in Symmetric spaces, ed. W. Boothby and G. Weiss, Marcel Dekker, New York, 1972.

[11] A. Korányi and M. A. Picardello, Boundary behaviour of eigenfunctions of the Laplace operator on trees, Ann. Scuola Norm. Sup. Pisa C1. Sci. (IV), 13 (1986), 389-399.

[12] H. L. Michelson, Fatou theorems for eigenfunctions of the invariant differential operators on symmetric spaces, Trans. Amer. Math. Soc., 177 (1973), 257-274.

[13] P. Sjögren, Characterizations of Poisson integrals on symmetric spaces, Math. Scand., 49 (1981), 229-249.

[14] Fatou theorems and maximal functions for eigenfunctions of the Laplace-Beltrami operator in a bidisk. J. Reine Angew. Math., 345 (1983), 93-110.

[15] _ A Fatou theorem for eigenfunctions of the Laplace-Beltrami operator in a symmetric space, Duke Math. J., 51 (1984), 47-56.

[16] __ Une remarque sur la convergence des fonctions propres du laplacien à valeur propre critique, pp. 544-548 in Théorie du potentiel, ed. G. Mokobodzki and D. Pinchon, Lecture Notes in Mathematics 1096, Springer, 1984.

[17] Admissible convergence of Poisson integrals in symmetric spaces, Annals of Math., 124 (1986), 313-335.

[18] E. M. Stein, Boundary behavior of harmonic functions on symmetric spaces: Maximal estimates for Poisson integrals, Invent. Math., 74 (1983), 63-83.

Received November 10, 1986.

DePartment of Mathematics

Chalmers University OF TeChNology

UNIVERSITY OF GÖTEBORG

S-412 96 GOTEBORG

SWEDEN 



\section{PACIFIC JOURNAL OF MATHEMATICS EDITORS}

\author{
V. S. VARADARAJAN \\ (Managing Editor) \\ University of California \\ Los Angeles, CA 90024 \\ HERBERT ClEMENS \\ University of Utah \\ Salt Lake City, UT 84112 \\ R. FINN \\ Stanford University \\ Stanford, CA 94305
}

\author{
HERMANN FLASCHKA \\ University of Arizona \\ Tucson, AZ 85721
}

RAMESh A. GANGolli

University of Washington Seattle, WA 98195

VAUGHAN F. R. JONES

University of California

Berkeley, CA 94720

\author{
ROBION KIRBY \\ University of California \\ Berkeley, CA 94720
}

C. C. MOORE

University of California

Berkeley, CA 94720

HAROLD STARK

University of California, San Diego

La Jolla, CA 92093

\section{ASSOCIATE EDITORS}
R. AREnS
E. F. BECKENBACH
B. H. NEUMANN
F. WOLF
K. YOSHIDA

(1906-1982)

\section{SUPPORTING INSTITUTIONS}

\begin{abstract}
UNIVERSITY OF ARIZONA
UNIVERSITY OF BRITISH COLUMBIA

CALIFORNIA INSTITUTE OF TECHNOLOGY

UNIVERSITY OF CALIFORNIA

MONTANA STATE UNIVERSITY

UNIVERSITY OF NEVADA, RENO

NEW MEXICO STATE UNIVERSITY

OREGON STATE UNIVERSITY
\end{abstract}

\author{
UNIVERSITY OF OREGON \\ UNIVERSITY OF SOUTHERN CALIFORNIA \\ STANFORD UNIVERSITY \\ UNIVERSITY OF HAWAII \\ UNIVERSITY OF TOKYO \\ UNIVERSITY OF UTAH \\ WASHINGTON STATE UNIVERSITY \\ UNIVERSITY OF WASHINGTON
}

The Supporting Institutions listed above contribute to the cost of publication of this Journal, but they are not owners or publishers and have no responsibility for its content or policies.

Mathematical papers intended for publication in the Pacific Journal of Mathematics should be in typed form or offset-reproduced (not dittoed), double spaced with large margins. Please do not use built up fractions in the text of the manuscript. However, you may use them in the displayed equations. Underline Greek letters in red, German in green, and script in blue. The first paragraph must be capable of being used separately as a synopsis of the entire paper. In particular it should contain no bibliographic references. Please propose a heading for the odd numbered pages of less than 35 characters. Manuscripts, in triplicate, may be sent to any one of the editors. Please classify according to the scheme of Math. Reviews, Index to Vol. 39. Supply name and address of author to whom proofs should be sent. All other communications should be addressed to the managing editor, or Elaine Barth, University of California, Los Angeles, California 90024.

There are page-charges associated with articles appearing in the Pacific Journal of Mathematics. These charges are expected to be paid by the author's University, Government Agency or Company. If the author or authors do not have access to such Institutional support these charges are waived. Single authors will receive $\mathbf{5 0}$ free reprints; joint authors will receive a total of $\mathbf{1 0 0}$ free reprints. Additional copies may be obtained at cost in multiples of 50 .

The Pacific Journal of Mathematics is issued monthly as of January 1966. Regular subscription rate: $\$ 190.00$ a year (5 Vols., 10 issues). Special rate: $\$ 95.00$ a year to individual members of supporting institutions.

Subscriptions, orders for numbers issued in the last three calendar years, and changes of address should be sent to Pacific Journal of Mathematics, P.O. Box 969, Carmel Valley, CA 93924, U.S.A. Old back numbers obtainable from Kraus Periodicals Co., Route 100, Millwood, NY 10546.

The Pacific Journal of Mathematics at P.O. Box 969, Carmel Valley, CA 93924 (ISSN 0030-8730) publishes 5 volumes per year. Application to mail at Second-class postage rates is pending at Carmel Valley, California, and additional mailing offices. Postmaster: send address changes to Pacific Journal of Mathematics, P.O. Box 969, Carmel Valley, CA 93924.

PUBLISHED BY PACIFIC JOURNAL OF MATHEMATICS, A NON-PROFIT CORPORATION Copyright (C) 1988 by Pacific Journal of Mathematics 


\section{Pacific Journal of Mathematics}

\section{Vol. 131, No. 2 December, 1988}

Selman Akbulut and Henry Churchill King, Polynomial equations of immersed surfaces .................................... 209

Alberto Baider and Richard C. Churchill, The Campbell-Hausdorff group and a polar decomposition of graded algebra automorphisms ........2 219

Wayne C. Bell and John William Hagood, Separation properties and exact

Radon-Nikodým derivatives for bounded finitely additive measures . . . 237

Dennis J. Garity, James P. Henderson and David G. Wright, Menger

spaces and inverse limits ...............................249

B. Brent Gordon, Algebraically defined subspaces in the cohomology of a

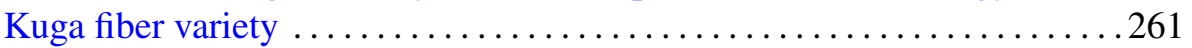

Jeffrey A. Hogan, Weighted norm inequalities for the Fourier transform on connected locally compact groups ........................... 277

Guojun Liao, A study of regularity problem of harmonic maps ..........291

Chin-pi Lu, Modules satisfying ACC on a certain type of colons ......... 303

Kunio Murasugi, Jones polynomials of periodic links

Hans Schoutens, Approximation properties for some non-Noetherian local

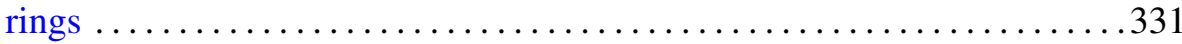

Peter Sjögren, Convergence for the square root of the Poisson kernel ...... 361 Alexandru Ion Suciu, The oriented homotopy type of spun 3-manifolds .393 